\title{
Emotionen der Skepsis
}

\author{
Von MARTIN HARTMANN (Frankfurt/M.)
}

\section{Der direkte Repräsentationalismus}

Stanley Cavell hat keine eigenständige Theorie der Emotionen entwickelt. Gleichwohl finden sich vor allem in seinen Shakespeare-Interpretationen gewichtige Anmerkungen über einzelne Emotionen, sodass es aufschlussreich sein mag, diese Anmerkungen in systematischer Absicht zu bündeln. Dabei soll es allerdings nicht darum gehen, aus diesen Anmerkungen eine vollständige Theorie der Emotion zu destillieren. Eher geht es darum zu zeigen, wie fruchtbar es sein kann, die Genese und das Wirken einzelner Emotionen in einem narrativen Kontext zu beleuchten. Auf diesem Wege gelangt man nicht zu einer Theorie der Emotionen und auch nicht zu einer vollständigen Theorie narrativer Emotionen, aber man gelangt zu Aussagen, die sich auf vorhandene Emotionstheorien beziehen lassen und sie an der einen oder anderen Stelle korrigieren oder bereichern. Wesentlich für diesen interpretatorischen Ansatz ist der Versuch, Cavells Denken, das nach wie vor wie ein großer fremder idiosynkratischer Block in der Landschaft der Gegenwartsphilosophie steht, an vorhandene Diskussionen heranzuführen, um auf diese Weise zu zeigen, dass ein Gespräch zwischen dieser eigentümlichen, wenn man so will: absolut individuellen Philosophie und anderen Denkschulen und Strömungen möglich ist. Inwieweit eine solche Übersetzungsarbeit dem Cavellschen Werk Gewalt antut, weil es aus dem Kontext zieht, was nur im Kontext seine irritierende Wirkung entfaltet, ist eine offene Frage, die wohl nur eine intensivere Beschäftigung mit Cavells Werk angemessen beantworten kann. Da die folgenden Überlegungen nur einen kleinen Winkel dieses Werks beleuchten, wird die Frage nach der Gewalt der Interpretation gar nicht erst aufkommen. Sie verfolgen offen die Absicht, einzelne Elemente der Cavellschen Shakespeare-Interpretationen aus ihrem Kontext herauszulösen und in einen anderen Kontext einzubetten, um zu untersuchen, inwieweit sie in diesem neuen Kontext bestehende Deutungsmuster stören können.

Der Kontext, um den es dabei geht, sei hier genannt. Emotionen können, so eine mittlerweile gängige Position, rational oder irrational, angemessen oder unangemessen, korrekt oder inkorrekt, begründet oder unbegründet oder sogar wahr oder falsch sein. Welche dieser Eigenschaften ihnen zugesprochen wird, ist naturgemäß nicht unerheblich, aber darauf soll es hier nicht ankommen. Emotionen besitzen diese Eigenschaften (die trotz ihrer internen Differenzen im Folgenden unter dem Begriff der Angemessenheit zusammengefasst werden), weil sie einen intentionalen und einen repräsentationalen Gehalt haben. Sie sind auf etwas gerichtet 
und stellen etwas dar. Wenn ich mich wegen eines Sprachfehlers schäme, richtet sich meine Scham auf etwas (meine Sprache) und stellt es auf bestimmte Weise dar (als beschämend, fehlerhaft, minderwertig und so weiter; oder als beschämend, weil fehlerhaft, minderwertig und so weiter). In beiden Hinsichten können wir die Emotion mit Blick auf ihre Angemessenheit oder Unangemessenheit beurteilen. Wir können sagen: Ein Sprachfehler ist ein möglicher Gegenstand der Emotion Scham, und uns dabei auf die Grammatik der Scham berufen, die Auskunft über die möglichen Gegenstände der Emotion gibt. Damit ist nicht gesagt, dass es richtig ist, sich wegen eines Sprachfehlers zu schämen, es ist nur gesagt, dass es möglich ist. Können wir uns in gleicher Weise wegen des Sprachfehlers eines anderen schämen? Ja, wenn wir mit dieser Person in enger Verbindung stehen (es handelt sich um den eigenen Sohn). Und wenn eine solche Verbindung nicht vorliegt? Können wir sagen ,Ich schäme mich, weil der Junge, den ich nicht kenne, stottert"? Dies scheint die spezifische Grammatik der Scham nicht zuzulassen, zu der ein Bezug auf Eigenes (und sei es die eigene Nation oder die eigene Mannschaft) gehört. Wir können darüber hinaus beurteilen, ob die Scham über den Sprachfehler repräsentational angemessen ist. Jemand könnte sagen ,Du brauchst dich nicht zu schämen, du stotterst doch gar nicht. ' Übersetzt in eine philosophische Sprache heißt das: Die Emotion hat den Ausschnitt der Welt, um den es geht, nicht angemessen dargestellt, ihr fehlt die Entsprechung in der Wirklichkeit, die sie gleichwohl als solche suggeriert.

Es ist dieser repräsentationale Gehalt von Emotionen, der dazu geführt hat, sie an Wahrnehmungen anzugleichen. Emotionen, so eine gängige Rede, lassen uns einen Gegenstand, eine Situation oder eine Person in einem bestimmten Licht sehen, in ihnen erscheint dieser Gegenstand, diese Situation oder diese Person so oder so. Hinzugefügt wird dabei zumeist, dass Emotionen ein spezifischer Wahrnehmungstyp sind, da wir in ihnen Wirklichkeit evaluativ sehen. Wenn ich einen Löwen sehe, sehe ich ein Tier mit bestimmten Eigenschaften; sehe ich diesen Löwen aber emotional oder im Lichte einer Emotion (etwa Furcht), dann sehe ich diese Eigenschaften als furchterregend und bleibe ihnen gegenüber nicht länger neutral. In der Emotion bewerte ich den Löwen als furchterregend oder nicht. Mehr noch, man kann sagen, dass ich die Eigenschaft, Furcht zu erregen, nur durch eine Emotion wahrnehmen kann, sodass den Emotionen eine irreduzible werterschließende Seite zukommt. Solange ich nicht emotional reagiere, sehe ich die furchterregenden Aspekte des Löwen nicht und kann sie auch gar nicht sehen. Weil uns Emotionen auf diese Weise evaluative Gehalte erschließen, führen wir sie auch an, um Handlungen oder handlungsrelevante Überzeugungen von uns zu rechtfertigen: ,Warum glaubst, dass der Löwe gefährlich ist?` - ,Ich habe es gespürt ${ }^{`}$ - das ist dann eine überzeugende Antwort, wenn man annimmt, dass die Emotion ein gutes oder sogar das einzige Mittel ist, um die Gefährlichkeit des Löwen in handlungsrelevanter Weise wahrzunehmen.

Bestandteil der Wahrnehmungsanalogie ist die Annahme, dass das, was sich uns in der Emotion evaluativ erschließt, an sich die evaluativen Züge besitzt, die die Emotion erschließt. Zwar lässt diese Formulierung noch offen, ob diese evaluativen Züge auch unabhängig von der Emotion vorhanden sind und durch sie gleichsam nur entdeckt werden oder ob sie in einem stärkeren Sinne an das Vorkommen der Emotion gebunden sind, aber egal, für welche Variante man sich entscheidet, es gilt, dass die in der Emotion erschlossenen evaluativen Gehalte keinen bloß subjektiven Status haben. In meiner Furcht vor dem Löwen erschließt sich der Löwe nicht nur als gefährlich für mich, sondern als gefährlich an sich. Nur weil die 
furchterregenden Eigenschaften einen die Emotion selbst transzendierenden Charakter haben, können wir emotionale Reaktionen kritisieren oder korrigieren. Mit anderen Worten, selbst wenn wir einräumen, dass es Emotionen gibt, deren Vorkommen konstitutiv für bestimmte Werte sind, hören wir nicht auf, nach Kriterien der Angemessenheit zu suchen, die über die Emotionen hinausgehen. ${ }^{1}$

Die Attraktivität der Wahrnehmungsanalogie liegt genau an diesem Punkt: So wie unsere Überzeugung, dass da ein Tisch ist, an unserer Wahrnehmung des Tisches hängt, so hängt die Überzeugung, dass jemand schuldig ist, an der Wahrnehmung der Situation als schuldrelevant. Und so wie wir Wahrnehmungen überprüfen können, wenn wir Zweifel an ihrer Richtigkeit haben, so können wir auch prüfen, ob eine Emotion das, was sie zu repräsentieren vorgibt, tatsächlich repräsentiert: „We tell“", so de Sousa, ,which [emotion] is wrong much as we test the veracity of perceptual information: by appealing to corroborating evidence. ${ }^{22}$ Emotionen, das ist eine der Lektionen der Wahrnehmungsanalogie, bringen uns in Kontakt mit Wirklichkeit, sie erweitern, in einer Formulierung McDowells, unser Gespür dafür, wie die Dinge sind (our sensitivity to how things are). ${ }^{3}$ Bezeichnet man diese Art der Bezugnahme auf Wirklichkeit als repräsentational, muss nur hinzugefügt werden, dass es sich um einen besonderen Typ repräsentationaler Bezugnahme handelt, nämlich um einen direkten im Gegensatz zu indirekten Typen. In der indirekten Bezugnahme beziehe ich mich auf dem Umweg über innere Zustände auf Wirklichkeit, sodass diese Zustände prinzipiell von dem, worauf sie verweisen, getrennt werden können. Meine Überzeugungen über den Mond setzen nicht voraus, dass der Mond gerade sichtbar ist oder überhaupt existiert (er existiert nur im Märchen). ${ }^{4}$ Emotionen dagegen können nicht in gleicher Weise von den Situationen oder Gegenständen, auf die sie verweisen, getrennt werden, jedenfalls dann nicht, wenn sie außerhalb fiktiver Kontexte thematisch werden. Der direkte Repräsentationalismus geht davon aus, dass Emotionen ihren repräsentationalen Gehalt immer in Reaktion auf reale evaluative Gehalte erhalten (sollten), sodass sie unmittelbarer mit Welt und mit Praktiken der Weltbewältigung verwoben sind, als das von anderen mentalen Phänomenen gesagt werden kann. Auch wenn die in Emotionen erschlossenen evaluativen Gehalte nicht vollständig unabhängig von menschlichen Praktiken existieren, gehen viele Ansätze davon aus, dass sich die in diese Praktiken eingelagerten Relevanzstrukturen immer auch von der Welt her ergeben oder sich von ihr her dem Subjekt aufdrängen: „We might say, particular emotions, as instances of our being attuned to import, are a kind of receptivity to that import, whereby import impresses itself upon us." ${ }^{\text {s }}$ Diese Rezeptivität der Emotionen für evaluative Gehalte passt gut zum häufig betonten passiven Charakter des emotionalen Lebens. Was sich uns in der Emotion als wichtig (oder gefährlich oder beschämend) erschließt, drängt sich uns auf, entspringt mithin nicht bewusster Wahl. Wir können nicht beschließen, uns zu schämen, sowenig wie wir beschließen können, uns zu freuen. Dass uns Emotionen zustoßen, passt folglich zu der Annahme, dass sich uns in ihnen etwas aufdrängt, dass wir in der Emotion immer auch passiv rezipierende Wesen sind. Aus diesen Überlegungen folgt, dass Emotionen dort, wo sie nicht auf die in ihnen unterstellten evaluativen Gehalte bezogen werden können, unangemessen sind und kritisiert werden können. Sie sind als mentale Phänomene involvierter, sind stärker eingebettet in eine von Relevanzen und Wichtigkeiten getragene Praxis. Genau deswegen bieten sie sich in den Augen vieler Autoren als ein direkterer Zugang zur Wirklichkeit an. 


\section{Scham, Eifersucht und Ekel in den Shakespeare-Deutungen Cavells}

Der direkte Repräsentationalismus, der damit umschrieben worden ist, zieht sich durch viele neuere Emotionstheorien. Er soll im Folgenden mithilfe der Shakespeare-Deutungen Cavells kritisiert werden. Es sind vor allem drei Annahmen des direkten Repräsentationalismus, die problematisch sind. Zum einen liefert der direkte Repräsentationalismus ein falsches Bild der Beurteilungsbasis menschlicher Emotionen, weil er suggeriert, Emotionen seien an sich angemessen oder unangemessen. Eine narrativ orientierte Theorie der Emotionen kann zeigen, dass diese Isolierung der Emotionen ihre Verwobenheit mit Selbstverständnissen unterschlägt und damit die eigentliche Basis unserer Beurteilung menschlicher Emotionen aus dem Blick gerät. Der direkte Repräsentationalismus unterstellt darüber hinaus, dass die Kriterien der Beurteilung menschlicher Emotionen einen die Emotionen transzendierenden Charakter haben. Die Wahrnehmungsanalogie impliziert, dass es etwas an der Wirklichkeit gibt, das uns dabei hilft, die Angemessenheit oder Unangemessenheit der Emotionen zu beurteilen. Dabei wird unterschlagen, dass alle mit Selbstverständnissen verwobenen Emotionen einen welterschließenden Zug haben, sodass es mit Blick auf Emotionen keine vollständig emotionsunabhängigen Kriterien der Beurteilung dieser Emotionen gibt. Das heißt nicht, dass Emotionen nicht angemessen oder unangemessen sein können; es heißt nur, dass die Kriterien der Beurteilung der Emotionen anders beschrieben werden müssen, als das im direkten Repräsentationalismus der Fall ist. Vor allem ihre normative Basis verschiebt sich, da die Unangemessenheit der Emotionen nicht länger ausschließlich epistemisch konzeptualisiert wird, sondern einen explizit ethischen Einschlag erhält. Der Cavellsche Anerkennungsbegriff liefert hier erste, wenn auch sehr grobe Bausteine einer ethischen Theorie der Emotionsbeurteilung. Schließlich neigt der direkte Repräsentationalismus dazu, die Frage nach dem methodischen Zugang zum Emotionsphänomen vollständig auszublenden, was in der Regel zu einem verengten Begriff der Emotionen führt. So bevorzugt der direkte Repräsentationalismus oft einen Emotionsbegriff, der sie auf aktuale affektive Zustände [states] reduziert und blendet damit dispositionale Emotionen per definitionem aus. Diese Reduzierung des Bereichs menschlicher Emotionen hängt mit uneingestandenen methodischen Vorannahmen zusammen. Die hier zu entfaltende Theorie narrativer Emotionen dagegen versucht von Anfang an zu klären, wie wir als Subjekte und als Beobachter von Emotionen den Phänomenbereich, um den es geht, für uns zugänglich machen. Indem sie von narrativ zu entfaltenden Selbstverständnissen ausgeht, schlägt sie vor, den Bereich menschlicher Emotionen als durch und durch interpretationsabhängig zu begreifen. Sie muss dabei nicht leugnen, dass Emotionen eine physiologische und eine phänomenale Seite haben, die nicht interpretationsabhängig sind. Sie behauptet nur, dass der Versuch, Emotionen zu verstehen, auf Interpretationen zurückgreifen muss, die aus der Perspektive der Ersten oder der Dritten Person angefertigt werden. Narrative sind in diesem Sinne Instrumente der Interpretation menschlicher Emotionen im Kontext eines Selbstverständnisses.

1. König Lears Scham. König Lear beschließt im Alter, sein Königreich unter seinen drei Töchtern, Goneril, Regan und Cordelia, aufzuteilen, um sich anschließend in ihre Obhut zu begeben. Um zu entscheiden, wie viel Land jede Tochter bekommen soll, verlangt er in der berühmten Abdankungsszene des ersten Aktes einen Liebesbeweis, den seine älteren Töchter, Goneril und Regan, auch eloquent darbringen. Seine Lieblingstochter allerdings, Cordelia, 
der er gerne das größte Stück Land gegeben hätte und die ihn wirklich liebt, verweigert sich dem unwürdigen Spiel, weil ihr nicht klar ist, wie sie angesichts der heuchlerischen Worte ihrer Schwestern in ihrer Liebe wahrhaftig erscheinen kann. Sie wird von Lear sofort ohne Land verbannt und verlässt Britannien mit ihrem zukünftigen Mann, dem König von Frankreich. Lear wiederum teilt das ganze Land unter seine beiden älteren Töchter auf und hofft, dass sie ihn abwechselnd pflegen werden. Doch er täuscht sich. Goneril und Regan verstoßen ihn von ihren Gütern und treiben ihn auf die Heide, wo ein Sturm wütet. Lear wird wahnsinnig. Seine einzige Begleitung sind der Narr, Kent (ein treuer Diener) und der als Tom von Bedlam verkleidete Edgar, der Sohn des Grafen von Gloucester, der sich auf Grund einer Intrige seines Halbbruders Edmund ebenfalls zur Flucht gezwungen sieht. Gloucester selbst hat den Zorn Gonerils und Regans auf sich gezogen, weil er Lear helfen will. Ihm werden die Augen ausgestochen. In einer berühmten Szene begegnen sich der blinde Gloucester und der zunächst noch wahnsinnige Lear. Lear erkennt Gloucester und erlangt langsam seine Vernunft wieder. Unterdessen hört Cordelia vom Schicksal ihres Vaters und drängt ihren Mann, in den Krieg gegen Britannien zu ziehen. Der Kampf geht verloren, Lear und Cordelia werden von Edmund gefangen genommen. Edmund befiehlt ihren Tod, Cordelia wird erhängt, Lear stirbt.

Noch bevor Cavell sich der Emotion der Scham zuwendet, um einige der wesentlichen Motivationen des Königs zu deuten, sind es andere Züge des Stücks, die sein Interesse hervorrufen. Wie kaum in einem anderen Stück Shakespeares nämlich kommt es im König Lear zu Szenen der Wiedererkennung, denen eine Weigerung, den anderen anzuerkennen, vorausgegangen ist. Lear verbannt Cordelia, weil sie ihm nicht den Überschwang der Liebe geschenkt hat, den er sich, so scheint es zumindest, von ihr erhofft hatte. „Ich liebe Eure Majestät gemäß meiner Schuldigkeit. Nicht mehr, noch weniger.“ (I, 2, 92-93) ${ }^{6}$ Es sind diese nüchternen Worte Cordelias, die Lears Wut hervorrufen und die fatale Entwicklung des Stücks in Gang setzen: „Fort, und bleib mir aus den Augen.“ (I, 2, 124) Was sich in dieser Szene ereignet, ist die Weigerung, Cordelia weiterhin als Tochter anzuerkennen, die einhergeht und sich symbolisiert in ihrer Verbannung aus dem Sichtkreis Lears. Auch Edgar, der Sohn des Grafen von Gloucester, sieht sich gezwungen, den Ort seiner Herkunft in dem Augenblick zu verlassen, in dem sein Vater ihn nicht länger in seiner wahren Natur erkennt und in ihm unter den Einflüsterungen Edmunds nur noch den „Schurken“ und „mörderischen Feigling“ wahrnimmt. Dass die Weigerung, die eigenen Kinder weiterhin als solche anzuerkennen, der Drang also, sie gleichsam ihres Status' als Kind und all der damit verbundenen Verpflichtungen zu berauben, einhergeht mit ihrer Verbannung, also ihrer Entfernung aus dem unmittelbaren Sichtkreis, ist sicherlich kein Zufall, verwendet Cavell den Begriff der Anerkennung [recognition] an dieser Stelle doch stets in seiner ganzen Vielschichtigkeit. In dem Maße, in dem Lear und Gloucester ihre Kinder nicht mehr als Kinder anerkennen, verlieren sie gleichsam das Vermögen, sie als ihre eigenen Kinder zu identifizieren, was sich spätestens in dem Augenblick zeigt, in dem sie ihre Kinder an den entscheidenden späteren Stellen des Stücks nicht nur wieder als ihre Kinder anerkennen, sondern zunächst überhaupt als die Individuen identifizieren, die sie sind: Cordelia und Edgar. In Lears „So wahr ich ein Mann bin, ich glaube, diese Dame ist mein Kind Cordelia“ (IV, 7, 68-69) drückt sich nicht nur die Tatsache aus, dass der langsam aus seinem Wahn erwachende König eine Person, die visuell schon länger für ihn sichtbar ist - er nennt sie in einer ersten Ansprache (IV, 7, 49) einen „Geist“ [spirit], 
dann schließlich „Dame“ -, endlich als seine Tochter Cordelia identifiziert und damit wiedererkennt, sondern auch und zugleich die Tatsache, dass er sie als sein Kind anerkennt, sie also nicht nur identifizierend wiedererkennt, sondern auch in ihren Status anerkennt. Dabei sollte deutlich sein, dass es sich hier nicht um zwei kognitive Schritte handelt, die nacheinander vollzogen werden. Es ist nicht so, dass Lear die „Dame“ zunächst als seine Tochter Cordelia identifiziert und dann als sein Kind anerkennt. Das identifizierende (Wieder)Erkennen und das bestätigende Anerkennen vollziehen sich vielmehr in einem Akt und können auch nur deswegen in einem Akt zerstört werden. ${ }^{7}$ Freilich wird mit diesen Überlegungen nicht ausgeschlossen, dass es in anderen Fällen ein identifizierendes Erkennen geben kann, das nicht mit Formen einer bestätigenden Anerkennung verbunden ist.

Mit diesen ersten Vorüberlegungen ist allerdings noch gar nicht die eigentliche Pointe der Cavellschen Verwendung des Anerkennungsbegriffs erfasst. In einem nächsten Schritt seiner Argumentation nämlich wendet sich Cavell der Frage zu, wie es überhaupt dazu kommt, dass die Protagonisten des König Lear die Anerkennung ihrer eigenen Kinder verweigern. Seine Antwort lautet, dass es zu den Vorbedingungen der Anerkennung anderer gehört, sich selbst anzuerkennen und im gleichen Schritt von anderen anerkennen zu lassen: „In each case, there is a condition necessary in order that the recognition take place: Gloucester and Lear must each first recognize himself, and allow himself to be recognized, revealed to another." ${ }^{\text {" }}$ Weil die bestätigende Anerkennung eines anderen nur in der Gegenwart dieses anderen vollzogen werden kann (also öffentlich), muss die Anerkennung schon in dem Augenblick scheitern, in dem sich das potenziell anerkennende Subjekt aus dem Sichtkreis des anderen entfernt und sich gleichsam vor ihm verbirgt. Genau hierin liegt für Cavell die Quelle der tragischen Entwicklungen des König Lear: In dem Maße, in dem weder Lear noch Gloucester sich selbst anerkennen, entziehen sie sich den Blicken Cordelias und Edgars, die, wie sie wohl wissen, aus nichts anderem als liebender Anerkennung bestehen. Die Verbannung ihrer Kinder, die auf den ersten Blick wie ein expliziter Akt einer einseitig verweigerten Anerkennung aussehen muss, besitzt folglich eine komplexere Struktur. Ihre eigentliche Motivation ist nicht so sehr die enttäuschte Liebe zweier Väter zu ihren Kindern, die sich in einem grausamen Akt des Liebesentzugs verdichtet, als vielmehr der untergründige Wunsch, von den Kindern nicht länger anerkannt (und das heißt immer auch: nicht länger gesehen) zu werden. Dieses Vermeiden von Liebe wiederum speist sich aus einem offenkundig gestörten Selbstverhältnis, für dessen genauere Beschreibung Cavell nun in einem weiteren Schritt seiner Argumentation den Begriff der Scham oder genauer: der Furcht vor Scham, die selbst schon schamgeleitet ist, in Anschlag bringt.

Es ist bekannt, und auch Cavell weist auf diesen Punkt hin, dass die Emotion der Scham mit dem Wunsch verbunden ist, den Blicken anderer verborgen zu bleiben. ${ }^{9}$ Dieser Wunsch wiederum entspringt zumeist dem Eindruck, bestimmten Erwartungen oder Normen, von denen man annimmt, dass sie allgemein geteilt werden, nicht entsprochen zu haben. Kennzeichnend für die Scham ist weiterhin, dass man sich weniger der eigenen Taten als vielmehr des eigenen So-Seins wegen schämt, das überhaupt dazu führte, die verworfenen Taten zu begehen. Der, der sich schämt, schämt sich, der zu sein, der er ist, und meidet folglich eine Anerkennung, die er nicht verdient zu haben glaubt. Diese Scham, das macht die eigentümliche Struktur der Scham aus, manifestiert sich dabei vor allem in einem Meiden der Blicke anderer, die, so die Befürchtung, sonst sehen könnten, was nicht gesehen werden soll. Es ist, mit anderen Worten, die Furcht vor dem Offenbarwerden der eigenen Unzulänglichkeiten, die den 
Wunsch nach Unsichtbarkeit hervorbringt. Wer sich schämt, nimmt gewissermaßen imaginativ vorweg, was gesehen werden könnte, wenn die eigene Verborgenheit verloren ginge, und reagiert auf diese Vorwegnahme mit Handlungen des Verbergens. Er sieht gewissermaßen in der Gegenwart, was andere zukünftig sehen könnten, sodass seine gegenwärtige Scham immer auch eine Furcht vor Scham ist. Cavell geht etwa davon aus, dass sich Gloucester der Herkunft seines unehelichen Sohns Edmund schämt - er nennt ihn humorvoll „Hurensohn“ (I, 1, 24) und ihn deswegen gerne fern von sich hält: „Er ist neun Jahre draußen gewesen, und weg soll er auch wieder.“ (I, 1, 32) Lears Scham dagegen ist komplexer und wird mithilfe unterschiedlicher Motivstränge von Cavell beschrieben. Lear fühlt sich der Liebe anderer unwürdig, da er ohne seine Macht gleichsam impotent dasteht; er vermeidet diese Liebe, um die in ihr zum Ausdruck kommende Bedürftigkeit, Passivität und Hilflosigkeit zu leugnen; er verlangt in der Abdankungsszene unechte, bloß gespielte Liebe von seinen Kindern, um auf vermeintlich echte Liebe nicht mit eigener Liebe reagieren zu müssen. Letztlich geht es ihm darum, die mit Liebe verbundenen Forderungen und Erwartungen nicht einlösen zu müssen. ${ }^{10}$ Dass Cavell sich nicht auf eines dieser Motive festlegen will, hat womöglich auch mit dem zu tun, was er den willkürlichen Charakter der Scham nennt: „It is familiar to find that what mortifies one person seems wholly unimportant to another: Think of being ashamed of one's origins, one's accent, one's ignorance; one's skin, one's clothes, one's legs or teeth. [...] It is the most isolating of feelings." ${ }^{11}$ Die Normen oder Erwartungen, die der von Scham Gebeugte verinnerlicht hat, müssen insofern nicht allgemein geteilt werden, es reicht, wenn sie der imaginierte Zuschauer teilt, dessen vorgestellter Blick im Voraus Scham verursacht.

Die Rolle dieser Motivschichten, soviel dürfte deutlich geworden sein, ist die, Lears Scham mit Gehalt zu versehen. Auffällig ist dabei, dass diese Scham die Verborgenheit sucht, indem sie die Welt, deren Blicke sie fürchtet, zerstört oder verbannt, und nicht, indem sie sich stumm zurückzieht. Cavell lässt darüber hinaus keinen Zweifel daran, dass Lears Scham auch dann noch fortwirkt, als er Cordelia am Ende des Stücks wiedererkennt und im Wiedererkennen anerkennt. Der Rückzug in die Gefängniszelle („Komm, lass uns fort zum Gefängnis; wir beide allein wollen singen wie Vögel im Käfig; [...] als ob wir Kundschafter der Götter (God's spies) wären“, V, 3, 8 f.) vollzieht noch einmal Lears Wunsch danach, zu „schauen, ohne gesehen zu werden“, zu lieben, ohne in der Welt Platz für diese Liebe zu machen. ${ }^{12}$ Mehr noch, auch Lears Wahnsinn muss als Versuch gedeutet werden, die Welt auf Abstand zu halten, und steht damit ein für eine Erfahrung des Weltverlusts, die in Cavells Augen sonst nur noch dem Skeptiker vertraut ist. Zugleich offenbart sich in diesem Wahn auf untergründige Weise Lears Liebe zu Cordelia, die er doch als solche zu leugnen scheint. In dem Maße, in dem Lear diese Liebe - seine ,höchste und letzte Sorge“ [ultimate care $]^{13}$ - auf Grund seiner Scham leugnen muss, zerstört er die Welt und ruft das Chaos herbei, durch das die Verwirklichung dieser Liebe unmöglich wird. Sein Wahn hilft ihm dabei, sein Wissen um diese Liebe zu verleugnen oder für sich zu behalten und nicht im Austausch mit seiner Welt zu testen oder zur Anwendung zu bringen. ${ }^{14}$ Sein Wahn ist ein Versuch, sich seines Wissens zu entledigen.

Gibt es an dieser Scham etwas Ungewöhnliches? Es sind zwei Dinge, die hier ins Auge fallen. Zum einen nutzt Lear seine Scham gewissermaßen, um sich von den Forderungen der Liebe und Anerkennung (des anderen) zu befreien. Geht man davon aus, dass diese Emotion schon die frühe Abdankungsszene strukturiert, dann kann diese als eine Inszenierung der Scham verstanden werden, die darauf aus ist, echte Bekundungen reziproker Liebe unmöglich zu machen. Lear begibt sich in der Abdankungsszene in die Rolle eines Zuschauers, der 
seinen Töchtern dabei zusieht, wie sie ein Stück aufführen, das den Titel Inszenierungen der Liebe trägt. Er zerstört damit die Bedingungen reziproker Anerkennung, da die Theatralisierung der Liebe die Gegenwärtigkeit durchkreuzt, die Voraussetzung gegenseitiger Anerkennung ist. Der bloß zuschauende Lear verwandelt seine Liebe zu Cordelia und ihre Liebe zu ihm, an denen er doch eigentlich nicht zweifelt, in etwas, was bewiesen (und erkannt) werden muss, zugleich aber im Rahmen einer theatralen Inszenierung gar nicht bewiesen werden kann, und hält so die Liebe fern von sich. Selbst wenn dieses Fernhalten der Liebe getragen ist von der Annahme, diese Liebe nicht zu verdienen, und somit auf einer als unmöglich zu verwirklichenden Sehnsucht nach Liebe beruht, bleibt es dabei, dass es die Scham ist, die das Leugnen dieser Bedürftigkeit möglich macht und damit die Wirklichkeit magisch verzerrt. Sie rechtfertigt gleichsam den Verzicht auf Anerkennung und die Weigerung, anerkannt zu werden.

Ein zweiter Punkt: Indem Cavell Emotionen in literarischen Stücken thematisiert, teilt er die Auffassung, dass sie eine narrative Struktur haben. Wollen wir Lears Verhalten verstehen, wollen wir uns seine Scham durchsichtig machen, müssen wir versuchen, sie, soweit es geht, in ihrer Genese darzustellen. Wir verfügen in König Lear zwar nicht über eine Narration, die sich auf das gesamte Leben Lears erstreckt, aber wir erhalten einen Ausschnitt, der einige entscheidende Wendepunkte seines emotionalen Lebens vor Augen führt. Wenn wir von Lears Scham sprechen, meinen wir nicht nur diesen oder jenen Augenblick, in dem sich seine Scham manifestiert (diese Augenblicke gibt es natürlich, und sie sind wichtig, um die darunter liegende Disposition als eine der Scham zu identifizieren), wir meinen die Scham, die sein Verhalten insgesamt erklärt. Cavells Interpretation dieses Stücks ermöglicht aber noch eine weitere Einsicht. Weiter oben hieß es, Narrative seien Methoden, um Emotionen in der Außenund in der Binnenperspektive zu verstehen. Mit anderen Worten, wir können auf die narrative Methode zurückgreifen, wenn wir Emotionen als Theoretiker rekonstruieren wollen, wir können aber auch auf diese Methode zurückgreifen, wenn wir uns selbst in der Betroffenenperspektive klar machen wollen, was eine Emotion bedeutet. Diese sehr allgemeine Bemerkung darf aber nicht darüber hinwegtäuschen, dass es Bedingungen des Erzählens gibt, die bestimmen, ob ich überhaupt in der Lage bin, meinen Emotionen narrativ zu begegnen, also sie narrativ zu erschließen. Diese Bedingungen wiederum können durch die Effekte einzelner Emotionen zerstört werden, sodass etwa ein Charakter wie Lear in seinem schambedingten Wahn das Vermögen verliert, seinen eigenen Erfahrungen eine narrative Struktur zu geben. Dieser Verlust des Vermögens, sich selbst zu erzählen, kann nun seinerseits Gegenstand einer fiktiven Erzählung sein, die in diesem Fall den Titel König Lear trägt und die auch in dem Augenblick noch weitergeht, in dem Lear selbst in seine wahnsinnige Eigenwelt abtaucht. Damit ist eine Möglichkeit veranschaulicht, die man die Möglichkeit der Fremdheit zwischen der Emotion und der Narration der Emotion nennen kann; mehr noch, es sieht so aus, als könne das Vermögen subjektiver Narration unter dem Einfluss einer Emotion verloren gehen, sodass nicht jede Emotion aus der Sicht des Subjekts immer schon eine Emotion ist, die (von ihm selbst) erzählt werden kann. Was an diesem Punkt durch ein Stück wie König Lear veranschaulicht wird, ist folglich die bedingte Autonomie der Emotion gegenüber der Narration. Bedingt heißt hier nur, dass auch Emotionen, die destruktiv wirken, ihre Geschichte haben, an Bedingungen, Umstände und Selbstverständnisse geknüpft sind. Das Stück König Lear erzählt diese Geschichte und schafft eine (ästhetische) Distanz zwischen dem Erfahrungsraum der Figuren und dem der Zuschauer. ${ }^{15}$ Es zeigt damit als dramatische Erzählung, in welchem Maße das Unvermögen, sich zu erzählen, mit der Verbannung anderer einhergeht, 
denen man sich erzählen könnte oder die in der Lage wären, die eigene Geschichte zu erzählen. Sich anderen nicht zu zeigen, heißt, nicht zum Gegenstand ihrer Erzählungen werden zu können ${ }^{16}$; es heißt auch, kein Gegenüber für die eigene Erzählung zu haben. In diesem Sinne zerstört Scham den Bezug des Subjekts auf seine Welt und Mitwelt und das Vermögen des Erzählens. In seinem Wahn ist Lear nicht länger in der Lage, eine Welt vorauszusetzen, auf deren Frage er eine Antwort geben müsste. Umso bezeichnender ist es, dass der Wiedererkennung Cordelias eine Frage vorausgeht, die Lear, wenn auch verzögert, zu verstehen scheint: „Sir, kennt Ihr mich?“ (IV, 7, 48)

2. Othellos Eifersucht. Othello, der „Mohr“, ist Feldherr der Armee von Venedig und mit der schönen Desdemona verheiratet. Sein Fähnrich, Jago, hatte gehofft, von Othello zum Leutnant befördert zu werden, muss aber zusehen, wie dieser Posten an Cassio geht. Jago will sich rächen und bedient sich dabei des venezianischen Edelmanns Roderigo. Dieser verwickelt Cassio in einen Streit, sodass Cassio Othellos Zorn auf sich zieht. Jago überredet Cassio, bei Desdemona um Vermittlung zu bitten, verfolgt dabei aber nur die Absicht, Othellos Eifersucht zu erregen. Er überzeugt Othello davon, dass Cassio ein Verhältnis mit Desdemona hat. Wichtigstes Indiz für Othello ist ein Taschentuch, das Jagos Frau, Emilia, gefunden hat. Jago entreißt es ihr, jubelt es Cassio unter, und als Othello das Taschentuch in Cassios Händen sieht, ist er vom Ehebruch überzeugt. Er tötet Desdemona, wird von Emilia über die wahren Hintergründe aufgeklärt und ersticht sich schließlich selbst.

Wie König Lear unter Bezug auf die Emotion der Scham, so wird Othello unter Bezug auf die Emotion der Eifersucht entschlüsselt. Auf den ersten Blick scheint hierin auch kein Problem zu liegen. Othello glaubt den Einflüsterungen Jagos und geht bald davon aus, dass ihn seine Frau Desdemona mit Cassio betrügt. Die normale Grammatik der Eifersucht hat ungefähr folgende Struktur: Ich fürchte den Verlust einer mir wichtigen Person durch eine dritte Person, der ich entsprechend mit Neid begegne. Wie diese einfache Begriffsbestimmung schon zeigt, setzt sich die Eifersucht aus mehreren Empfindungen zusammen (Furcht, Neid) und beschreibt folglich weniger eine eigene phänomenal eindeutig ausweisbare Emotion als vielmehr eine spezifische Konstellation einzelner Emotionen. Hinzu kommen noch die Empfindungen, die dem geliebten Wesen gelten, dessen Verlust droht. Spinoza hat gerade diesen Punkt hervorgehoben: „Wenn sich jemand vorstellt (si quis imaginatur), daß der geliebte Gegenstand sich mit einem anderen durch ein gleiches oder engeres Band der Freundschaft verbindet, als das war, wodurch er ihn allein in Besitz hatte, so wird er von Haß gegen den geliebten Gegenstand affiziert werden und jenen anderen Gegenstand beneiden.“ In der Anmerkung zu diesem Lehrsatz heißt es dann: „Dieser Haß gegen den geliebten Gegenstand, der mit Neid verbunden ist, heißt Eifersucht. "17 Dass es sich hier um eine Konstellation einzelner Emotionen handelt, erklärt auch, warum es nicht nur möglich ist, auf eine Person eifersüchtig zu sein, sondern auch auf eine Beziehung oder ein Verhältnis zwischen Personen. Das intentionale Objekt der Eifersucht ist nicht so spezifisch, wie das bei anderen Emotionen der Fall ist. Generell aber gilt: Der Eifersüchtige will den nicht verlieren, auf den er ein Besitzrecht zu haben glaubt. Er ist neidisch auf den, der in seinen Augen geneigt ist, diesen Anspruch zu bestreiten, weil er spürt, dass die Gewinne, die der andere für sich reklamieren kann, seine Verluste sind, und wütend oder voller Ressentiment gegenüber dem geliebten Partner, von dem er vermutet, dass er eine bislang als exklusiv empfundene Intimität 
einem anderen zugänglich macht. Die genauere kognitive Struktur der Eifersucht ist dabei kompliziert. Der Eifersüchtige ist sich der absoluten Berechtigung seiner Eifersucht nie gewiss, aber seine Eifersucht treibt ihn im gleichen Maße dazu an, diese Gewissheit zu suchen, wie sie ihr Eintreten fürchtet. Der Eifersüchtige neigt dazu, nach Bestätigungen zu suchen, die seine Emotion als berechtigt ausweisen, und er findet diese Bestätigungen überall. Dabei wäre es zu einfach, darauf hinzuweisen, dass diese Beweise Zeichen einer irrationalen affektiven Wahrnehmung sind, die Beweise sieht, wo keine Beweise sind. Auch wenn dies durchaus der Fall sein kann, ist nicht ausgeschlossen, dass der Eifersüchtige Hinweise entdeckt, die aus einer Sicht als Rechtfertigung der Eifersucht dienen können. Tritt dieser Fall ein, hat sich der Eifersüchtige gleichsam im Sinne einer self-fulfilling prophecy die Beweise verschafft, die seine Eifersucht überhaupt erst als berechtigt erscheinen lassen. Während die Scham eher den Rückzug sucht oder, wie im Falle Lears, die Entfernung der anderen, die nicht sehen sollen, nicht anerkennen sollen, was zu sehen und anzuerkennen ist, verfügt die Eifersucht über investigative Züge und ist damit die aktivere Emotion. Nicht anders als die Scham aber ist auch die Eifersucht nicht selten getragen von der Kraft der Vorstellung (,si quis imaginatur“). Der Eifersüchtige imaginiert den geliebten Partner in den Armen des anderen, so wie der von Scham Gebeugte die Blicke anderer imaginiert, die nicht real anwesend sein müssen. Imaginiert er sich selbst im gleichen Atemzug als den „Hahnrei“, kann es zu einer eigentümlichen Verflechtung von Eifersucht und Scham kommen. In das Bild der in den Armen des anderen versinkenden geliebten Partnerin können Zuschauer integriert sein, die sich über mich als betrogenen Ehemann lustig machen. Nicht zuletzt dieser potenziell beschämende Aspekt der Eifersucht dürfte für ihren unangenehmen hedonischen Charakter verantwortlich sein. Es lässt sich kaum bezweifeln, dass die Eifersucht als Emotion nicht erstrebenswert ist. Niemand wird gern betrogen, niemand verliert gern ein geliebtes Wesen. Und das gilt auch dann, wenn die Eifersucht unberechtigt ist. Als Emotion zählt sie zweifellos zu den Emotionen, die zwar insofern positiv sein können, als sie uns Auskunft über das geben, was uns wichtig ist ${ }^{18}$, aber es fällt schwer, diesen positiven Aspekt ausschließlich positiv zu sehen, weil sich der andere in der Eifersucht als jemand darstellt, der uns verloren gehen kann.

Behält man diese Punkte im Auge, fällt an Cavells Deutung Othellos ein erster ungewöhnlicher Zug auf. Cavell geht nämlich davon aus, dass Othello das Betrogenwerden durch Desdemona förmlich sucht und begehrt: „Ich sage, daß wir [...] Othello so verstehen müssen, daß er Jago glauben will, daß er, wider sein besseres Wissen, ihm zu glauben versucht." ${ }^{19}$ Aber warum sollte er das tun? Warum sollte er Desdemona als untreu erkennen wollen? Und warum sollte er das tun wollen, obwohl er, wie Cavell an dieser Stelle ja unmissverständlich sagt, vom Gegenteil überzeugt ist? Die entscheidende Frage an diesem Punkt ist die nach den Gründen, die Othello hat, um ein Wissen, über das er verfügt, nicht als ein solches anzuerkennen. Was weiß Othello, ohne es wissen zu wollen? Und welchen Dienst leistet seine Eifersucht in diesem Prozess der Verleugnung seines Wissens? Die bündige Antwort, die Cavell auf diese Fragen liefert, lautet so: Othello weiß, dass „Desdemona existiert, daß sie Fleisch und Blut ist, getrennt von ihm und jemand anderes“. ${ }^{20}$ Und es ist, so heißt es an dieser Stelle weiter, genau diese Möglichkeit, „die ihm Qualen bereitet. Der Inhalt seiner Qual ist das Gefühl, daß ein anderer existiert, folglich auch er selbst existiert, und zwar als abhängig, als ein Teil.“ Wie unschwer zu erkennen ist, verknüpft Cavell auch in seiner Deutung Othellos eine Reihe von Motivschichten, die nicht leicht zu entwirren sind. So geht Cavell etwa davon aus, dass Othello Desdemona zur Frau wählt, weil sich in ihr seine eigene Reinheit spiegeln 
soll, weil er in ihr sein reines, edles Gesicht sehen will. Auf diese Weise gibt ihm Desdemona Zutrauen „zum eigenen Bild“. ${ }^{11}$ Genau dieses Bild wird nun in der Deutung Cavells durch Desdemonas Leiblichkeit und Sexualität, durch ihr Fleisch-und-Blut-Sein beschmutzt, und zwar unabhängig von der Frage, ob Othello selbst oder ein anderer den sexuellen Akt mit ihr vollzogen hat. Othellos „Logik“ sieht hier, in den Worten Cavells, so aus: „Entweder habe ich ihr [jungfräuliches] Blut vergossen, oder ich habe es nicht getan. Wenn nicht, war sie keine Jungfrau, und das befleckt mich. Wenn doch, ist sie nicht länger eine Jungfrau, und das befleckt mich.“22 Die Eifersucht ist nun für Othello unter anderem deswegen interessant, weil sie gleichsam ein Skript zur Verfügung stellt, in dem er Desdemona und einen anderen für die Befleckung verantwortlich machen kann, sodass er selbst seine Reinheit behält. Damit ließe sich die Fleischlichkeit Othellos, die Desdemona offensichtlich zu reizen vermochte, weiterhin leugnen. Dieses psychologische Motiv erklärt bereits, wie es möglich sein kann, dass eine an sich unangenehme Emotion wie die Eifersucht Teil eines Narrativs werden kann, in dem sie eine ablenkende und für das Subjekt durchaus positive Rolle erfüllt. Dennoch bleibt natürlich etwas Eigentümliches an der Deutung Cavells bestehen. Es bleibt ja dabei, dass Aspekte wie die Abhängigkeit vom anderen oder die Angewiesenheit auf ihn in der Regel in der Eifersucht zum Vorschein kommen, nicht aber von ihr verleugnet werden. Im Rahmen der ,,normalen" Grammatik der Eifersucht ist diese Emotion schlicht die Kehrseite und Begleiterscheinung der Liebe oder anderer Formen der Nähe, damit aber solange unausweichlich, wie uns an Liebe und Nähe mit ihren Exklusivitätsansprüchen etwas liegt. ${ }^{23}$ Wie gelangt Cavell von hier zu der Behauptung, in der Eifersucht könne Abhängigkeit geleugnet werden?

Es ist, wenn man so will, eine kleine Verschiebung in der Interpretation des EifersuchtsBegriffs, die diese eigentümliche Lektüre ermöglicht. Im Kontext der „,normalen“ Grammatik der Eifersucht fürchten wir in der Eifersucht um den Verlust des geliebten Wesens und erkennen damit untergründig an, dass dieses Wesen auf eine ganz fundamentale Weise von uns getrennt ist, eine Weise, die als Voraussetzung oder Bedingung der Möglichkeit konkreter Trennungen zu verstehen ist. Diese in der Emotion enthaltene Anerkennung allerdings, die vor allem Nussbaum zur Grundlage ihrer Emotionstheorie gemacht hat, ist in der Regel individuell gebrochen und wird entsprechend als Furcht vor dem Verlust eines konkreten anderen erfahren. In diesem Sinne richtet sich die Eifersucht zumeist auf die Möglichkeit einer solchen konkreten Trennung, nicht aber auf das Faktum der fundamentalen Getrenntheit des anderen an sich. Genau das aber ist, nach Cavell, bei Othello der Fall, der damit dem Begriff der Eifersucht eine andere Wendung gibt. Othellos Eifersucht richtet sich auf die „bloße Existenz“ des anderen, er sucht einen Besitz, der niemals von anderen streitig gemacht werden kann: „He seeks a possession that is not in opposition to another's claim or desire but one that establishes an absolute or inalienable bonding to himself, to which no claim or desire could be opposed, could conceivably count. ${ }^{24}$ Othellos Problem ist nicht so sehr Desdemonas vermutete Untreue, es ist vielmehr ihre bloße Existenz als solche, ihre Getrenntheit von ihm, die ihn belastet. Wenn das aber so ist, dann verleugnet Othellos Eifersucht gerade das, was in ihr zugleich zum Ausdruck kommt: die Getrenntheit und Unkontrollierbarkeit des anderen. Sie verleugnet es, indem sie es skandalisiert und moralisiert, indem sie daraus eine Art strafwürdiges Fehlverhalten des anderen macht und so suggeriert, es könne auch anders sein, mithin suggeriert, der andere könne sich entscheiden, nicht getrennt und nicht unkontrollierbar zu sein, könne den Entschluss fassen, aus der intersubjektiven Zweiheit eine unauflösliche Einheit zu machen. Diese Getrenntheit aber ist ein Faktum, an dem 
sich nicht rütteln lässt. Gleichwohl zeigt sich dieses Faktum, manifestiert sich, drängt sich auf. In Othellos Fall ist es vor allem Desdemonas eigenständige Sexualität, durch die sie für ihn zum separaten Handlungszentrum wird, dessen Begehren er, Othello, zu seinem eigenen Erschrecken weckt.

Damit liegt nun vor, was Othello weiß und verleugnet. Es kann dabei nicht überraschen, dass dieses Leugnen auch die Gestalt des Wissenwollens annimmt, etwa des Wissenwollens der Treue: „Schurke“, so sagt Othello zu Jago, ,sei sicher und beweise, daß meine Geliebte eine Hure ist, sei dessen sicher, gib mir den sichtbaren Beweis." (III, 3, 366) ${ }^{25}$ Setzt man voraus, dass Othello letztlich um die Treue Desdemonas weiß, dann liegt hier ein Zug vor, dieses Wissen als Problem oder als begrenzt zu behandeln und genau damit die Anerkennung des anderen zu umgehen. Othellos Skepsis verbirgt damit seine Ablehnung zu wissen, was zu wissen ist: „Was diesem Mann gefehlt hat, war nicht Gewißheit. Gewußt hat er alles, aber er

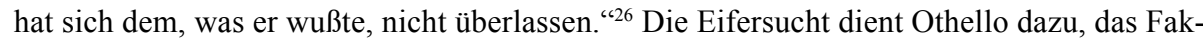
tum der Getrenntheit des anderen als kontingent zu behandeln, um es in gewisser Weise disponibel zu machen. Deswegen nimmt er Jagos Verdächtigungen bereitwillig auf, deswegen sucht er nach Beweisen für Desdemonas Untreue, als wäre das Faktum der Getrenntheit etwas, was sich beweisen ließe, und damit eben auch etwas, für das eventuell keine Beweise gefunden werden können. ${ }^{27}$

3. Leontes' Eifersucht. Leontes, König von Sizilien, unterstellt seiner schwangeren Frau, Hermione, ein Verhältnis mit Polixenes, dem König von Böhmen, zu haben. Polixenes, der gerade auf Sizilien weilt, weil er der gemeinsame Freund von Leontes und Hermione ist, soll von Camillo, einem engen Vertrauten Leontes', ermordet werden, doch Camillo weiht Polixenes ein, und beide fliehen nach Böhmen. Unterdessen bezichtigt Leontes Hermione des Hochverrats und lässt sie einsperren. Sie bekommt im Gefängnis ein Mädchen, das Leontes jedoch an einer fremden Küste aussetzen lässt (tatsächlich wird sie ohne sein Wissen nach Böhmen gebracht). Zur Sicherheit lässt Leontes noch das Orakel von Delphi befragen, doch das Orakel spricht Hermione von aller Schuld frei. Als Leontes das Orakel nicht akzeptiert, erhält er die Nachricht, dass sein Sohn, Mamillius, gestorben ist. Anschließend wird ihm auch die Nachricht vom Tod Hermiones überbracht. Die zweite Hälfte des Stücks spielt in Böhmen. Dort wächst die Tochter von Leontes und Hermione, Perdita, bei Schafhirten auf. Der Sohn von Polixenes, Florizel, verliebt sich in sie, aber da die Beziehung von seinem Vater als unstandesgemäß eingestuft wird, fliehen Perdita und Florizel mithilfe Camillos nach Sizilien. Polixenes verfolgt ihn, sodass alle bald in Sizilien vereinigt sind. In Anwesenheit Leontes' wird schließlich die Identität Perditas geklärt, es kommt zur Versöhnung mit Polixenes. Zum Schluss präsentiert Pauline, eine Vertraute Hermiones, eine Statue, die aussieht wie Hermione. Die Statue erwacht zum Leben, das Stück endet glücklich.

Dass Hermione am Ende des Wintermärchens als Statue wiederkehrt und Leontes akzeptiert, dass sie aus diesem Zustand heraus dem Leben wiedergegeben wird, beschreibt für Cavell eine Folge des Skeptizismus, wie sie in Othello und Leontes veranschaulicht wird. Schon Othello hatte Desdemona „versteinert“ - „doch werde ich ihr Blut nicht vergießen und auch nicht diese ihre Haut verunstalten, die weißer ist als Schnee und glatt wie Grabalabaster“ (V, 2, 4-5) - und sie daraufhin getötet. Nicht anders als Othello nutzt Leontes ein gewisses „Eifersuchtstheater“28, um seine Verwiesenheit auf andere zu leugnen, um sich etwa der 
Schuldigkeiten des Vaterseins zu entledigen. Seine Eifersucht dient ihm dazu, die Treue seiner Frau in Zweifel zu ziehen, aber sie dient ihm darüber hinaus auch dazu, seine eigene Vaterschaft in Zweifel zu ziehen, indem ein anderer Vater ersonnen wird. Damit gewinnt sie erneut eine eigentümliche Rolle, wird sie doch nicht von der leisen Hoffnung gespeist, sie möge unberechtigt und ganz und gar gegenstandslos sein, sondern von der Hoffnung, sie möge wahr oder berechtigt sein. Wenn es Gerüchte gibt, wonach Leontes nicht der Vater seines Sohnes ist, dann ist der Gegenstand seiner Furcht die „Negation dieser Gerüchte“, und die besagt, „dass er der Vater ist“ ${ }^{29}$ Mit anderen Worten, Leontes möchte nicht, dass die Gerüchte falsch sind, denn dann ist er der Vater seines Sohnes. Mamilius' Tod, über den im Stück nicht weiter verhandelt wird, erfüllt dieser Lesart nach gerade den Wunsch Leontes', nicht der Vater seiner Kinder zu sein. Vorweggenommen wird er in einer Szene, in der er seinen Zweifeln Ausdruck verleiht: „Mamilius, bist du mein Junge?“ (I, 2, 118). ${ }^{30}$ Es folgt die Suche nach Beweisen, die belegen sollen, dass Mamilius sein Sohn ist, er vergleicht Nase und Kopfform, sucht nach Belegen der Ähnlichkeit, die ihn, wie nicht anders zu erwarten ist, kaum befriedigen. Denn wie Treue lässt sich auch Kindschaft jenseits moderner Testverfahren nicht zum Gegenstand eines Beweisverfahrens machen, sodass erneut der Eindruck entsteht, hier werde nach Beweisen für Unbeweisbares gesucht.

Aber warum Vaterschaft leugnen? Warum auch hier leugnen, was doch eigentlich gewusst wird? Stärker noch als in seinen anderen Interpretationen einzelner Figuren Shakespeares stellt Leontes für Cavell das Porträt des Skeptikers dar, der sein Unvermögen, andere anzuerkennen, in die Form einer Unwissenheit kleidet, die sich durch mehr Wissen beheben lassen soll. Auch dieses Unvermögen aber führt letztlich auf das Leugnen menschlicher Endlichkeit und Abhängigkeit, das im Wintermärchen nihilistische Züge annimmt. Leontes „,rächt“ sich an der Existenz, an den Fakten des Lebens, zu denen Bindungen, Vereinigungen und Zusammenschlüsse ebenso gehören wie Trennungen, Abschiede und Auflösungen. ${ }^{31}$ Diese Welt, diese endliche Welt entzieht sich dem Skeptiker, und sie entzieht sich, weil er sie nicht länger ertragen kann, weil nur dann, wenn es nichts gibt, die Möglichkeit der Trennung verloren geht, die Leontes mit Grauen füllt. Kinder, die tot sind, können sich nicht trennen, und das gilt auch von Frauen, die zu Stein geworden sind. Leontes' Theater der Eifersucht gibt ihm die Mittel an die Hand, seine Strategie der Weltvernichtung begründet umzusetzen, sie liefert ihm ein Skript, um Zweifel zu sähen, Nachforschungen anzustellen und Verbannungen auszusprechen. In der Eifersucht offenbart sich folglich nicht die Angewiesenheit auf den anderen, sondern gerade ihre Leugnung, weil sie zwar vorgibt, den anderen besitzen zu wollen, diese Vorgabe aber nur nutzt, um ihn zu verstoßen. Sie kann dies tun, weil sie, wie erwähnt, eine zusammengesetzte Emotion ist, in der Elemente wie Liebe und Verlustangst auf Elemente wie Neid und Ressentiment treffen. Nicht dass sie notwendig destruktive Züge annehmen müsste; sie kann dies aber tun, wenn sie mit einem Selbstverständnis verwoben ist oder von einem Selbstverständnis getragen wird, das - aus welchen Gründen auch immer - die Andersheit (der anderen) nicht hinnehmen kann, weil sie Schuldigkeiten impliziert, also Reziprozität, also Gefordertsein.

4. Coriolanus' Ekel. Das Stück Coriolanus spielt im frühen Rom. Der stolze Patrizier Coriolanus besiegt den schlimmsten Feind Roms, Aufidius und seine Volsker, in der Schlacht und soll als Lohn zum Konsul gewählt werden. Dem Kriegsheld aber fällt es schwer, dem von ihm verachteten Volk gegenüberzutreten, um sich als Konsul bestätigen zu lassen. Immer 
wieder dringt sein von Verachtung gefüllter Ekel vor dem Volk durch, sodass er schließlich durch den Einfluss der rhetorisch geschickten Volkstribunen Sicinius und Brutus unter Druck gerät und Rom verlässt. Er schließt sich der erneut vor Rom stehenden Armee der Volsker an und plant, seine Heimatstadt mit ihrer Hilfe zu vernichten. Erst nach Intervention seiner Mutter, Volumnia, gibt er diesen Plan auf. Das wiederum empfindet Aufidius, der Feldherr der Volsker, als Verrat; Coriolanus wird getötet.

Die bislang thematisierten Emotionen der Scham und der Eifersucht zeichnen sich dadurch aus, dass sie die Arbeit, die sie in den Interpretationen Cavells verrichten, nur im Rahmen eines spezifischen Narrativs verrichten. Weder Scham noch Eifersucht müssen per se die destruktiven Konsequenzen haben, die Cavell unter Bezug auf einzelne Stücke Shakespeares expliziert. Scham könnte meine Zugehörigkeit zu einer Kultur andeuten, deren Werte und Normen ich verinnerlicht habe; Eifersucht könnte meine Verwiesenheit auf einen anderen verraten, den ich nicht verlieren will, in sie könnte also ein anerkennendes Moment einfließen. Dass weder die eine noch die andere Emotion diesen anerkennenden Zug tatsächlich aufweisen, ist bedingt durch die Tatsache, dass sie in ihrem konkreten Gehalt, und das heißt auch in ihren handlungsrelevanten Aspekten, getragen werden von einem Selbstverständnis, das bestimmt, wie die Welt unter dem Einfluss der Emotion wahrgenommen und praktisch relevant wird. Damit muss nicht geleugnet werden, dass Emotionen wie Scham und Eifersucht typische körperliche, phänomenale und behaviorale Eigenschaften aufweisen und dass die Art und Weise, in der Cavell diese Emotionen unter Bezug auf Shakespeare behandelt, atypische Züge trägt. Entscheidend für die hier angestellten Überlegungen aber ist einerseits, dass nicht nur die atypischen, sondern auch die typischen emotionalen Reaktionsweisen abhängig von Selbstverständnissen sind, und dass andererseits gezeigt werden kann, dass Elemente des Atypischen, auf wie untergründige Weise auch immer, in vielen typischen emotionalen Reaktionen vorhanden sind.

Im Unterschied zur Scham und zur Eifersucht fällt es schwer, im Ekel andere als typische Reaktionsweisen auszumachen. Auch wenn Ekel nicht frei ist von beweglichen ideellen oder kognitiven Elementen, scheint er in seiner Struktur unmittelbarer und direkter zu sein. Folglich drückt er in Cavells Interpretation von Coriolanus auch direkter aus, wie es um Coriolanus selbst steht. Während Lear, Othello und Leontes ihre Teilnahme am endlichen menschlichen Leben eher auf indirekte Weise leugnen, nimmt Coriolanus im Ausdruck seines Ekels direkte und unmissverständliche Distanzierungen und Leugnungen vor. Eine solche Deutung des Ekels stimmt überein mit anderen Deutungen, die aussprechen, was Cavell nur voraussetzt. Der Ekel vor Ausscheidungen aller Art, vor verfaultem Essen, verwesenden Körpern, vor Schimmel, Schleim und Kot hilft uns, unsere eigene Animalität und Sterblichkeit von uns fern zu halten, er schützt uns vor Verunreinigung und Beschmutzung. ${ }^{32}$ Anders als andere Emotionen also, die in ihrer typischen Ausprägung unsere Bedürftigkeit und Angewiesenheit auf andere anerkennen, leugnet Ekel, was doch gilt: dass wir sterbliche menschliche Wesen mit einem Körper sind, der verwest, wenn man ihn lässt. Ist diese Deutung des Ekels jedoch schlüssig, dann braucht Cavell in seinem Zugang zum ekelerfüllten Coriolanus offenbar einige der interpretatorischen Umwege nicht zu machen, die er in seiner Deutung der anderen ShakespeareStücke zweifellos gemacht hat. Es gehört gleichsam zum kognitiven Gehalt des Ekels, in ihm unbestreitbare Sachverhalte zu leugnen oder zumindest ihre Akzeptanz auf Distanz zu halten. ${ }^{33}$ Wenn also alle von Cavell thematisierten Emotionen ein Wissen bestreiten, dann tut der 
Ekel dies allein schon auf Grund seiner inneren Struktur und nicht erst als Element eines Narrativs, in dessen Rahmen die Emotionen die bestreitende Rolle erst gewinnen, die sie von sich aus nicht haben.

Dennoch ist es möglich, auch den Ekel des Coriolanus als Ausdruck oder Instrument eines Selbstverständnisses zu sehen. Das zeigt sich, wenn sein Ekel genauer eingekreist wird. Worauf richtet er sich? Und warum? Zunächst richtet sich sein Ekel auf das Volk, das er an verschiedenen Punkten beleidigt: ,abtrünnige Rüpel“ (I, 1, 159), „Kanaillen“ (I, 1, 164), „Krümel“ (fragments, I, 1, 217), ,niederes Hundepack, dessen Atemhauch ich hasse wie den Gestank fauler Sümpfe“" (III, 3, 121) - das sind nur einige der Flüche, die Coriolanus für das Volk bereit hält. ${ }^{34}$ Aber woraus speist sich diese Verachtung? Folgt man der Deutung Cavells, dann sind es verschiedene psychologische Schichten, die hier in der Persönlichkeit des Coriolanus aufeinander treffen und die sich in seiner Verachtung und seinem Ekel verdichten. Das Volk fordert am Anfang des Stücks Nahrung, da es hungert. Coriolanus beschimpft es dafür, als wäre das Verlangen, den Hunger zu befriedigen, Bestandteil einer niederen Natur. Dabei hat auch Coriolanus Hunger, aber, so Cavell, sein Hunger zielt darauf, keinen Hunger mehr zu haben, er begehrt, nichts mehr zu begehren: „Es ist besser zu sterben, besser zu verhungern, als nach dem Lohn zu schmachten (crave), den wir von Anfang an verdienen.“ (II, 3, 107) Das sagt Coriolanus in der Szene, in der er sich einzelnen Vertretern des Volks stellen muss, um für sich als Konsul zu werben. Etwas früher hat sich folgender Austausch ergeben:

Coriolanus. Ihr kennt den Grund, Herr, weswegen ich hier stehe.

3. Bürger. Das tun wir, Herr. Sagt uns, was Euch dazu gebracht hat.

Coriolanus. Mein eigenes Verdienst.

2. Bürger. Euer eigenes Verdienst?

Coriolanus. Ja, nicht mein eigenes Verlangen (desire).

(II, 3, 60 f.)

Der, der alles verdient, braucht nichts mehr zu wünschen, da ihm alles, was man sich wünschen könnte, immer schon (von Anfang an) zusteht. Indem er sich also für das Volk verdient macht, schafft er einen Zustand, der das Bitten und Werben um Anerkennung umgeht. Nicht anders als Leontes strebt auch Coriolanus in den Augen Cavells ein Überwinden des Menschlichen an, ein Überwinden eigener Sterblichkeit. Letztlich will er ein Gott werden. ${ }^{35}$ Sein Ekel schafft Distanz zum Volk, das in seinem Hunger Menschliches offenbart. Wirklich sprechen kann Coriolanus mit dem Volk nicht, weil Sprechen immer auch bedeutet, Bedürfnisse mitzuteilen und Kooperation zu ermöglichen. Der Zug zum Göttlichen kleidet sich für Cavell auch in die Parallelisierung von Coriolanus mit Christus. Wie Christus will Coriolanus das Volk mit Worten speisen und wundert sich, dass es diese Nahrung nicht annimmt. Wie Christus glaubt er sich zu opfern und leidet daran, dass die, für die er sich opfert, dieses Opfer nicht verdienen. Anstatt zu sehen, dass er das Volk nährt, mit Worten und mit seiner Tapferkeit, wirft es ihm vor, Nahrung zurückzuhalten. Als Konsequenz aus dieser empfundenen Undankbarkeit weigert sich Coriolanus, dem Volk weiterhin „Gutes“ (good words, I, 1, 163) mitzuteilen, ja, er weigert sich, Sprache mit ihm, dem Volk, zu teilen, weil sie etwas ist, was davon lebt, durch verschiedene Münder zu gehen: „A pervasive reason Coriolanus spits out words is exactly that they are words, that they exist only in a language, and that a language is metaphysically something shared, so that speaking is taking and giving in your mouth the very 


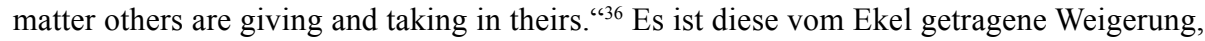
eine Sprache mit denen zu sprechen, die nichts verdienen, die ihn unfähig macht, das politische Leben zu lenken, bedeutet Politik doch notwendig Miteinander-Sprechen. Im Ekel distanziert er sich nicht so sehr von den Wünschen des Volks, die in seinen Augen niederer Natur sind, er distanziert sich davon, dass es Wünsche hat, dass es Abhängigkeit einräumt, ohne die mit ihr verbundene Reziprozität wirklich zu verdienen. Wirklich verstehen lässt sich sein Ekel folglich nur, wenn man weiß, als was er sich sieht und wie er sein Schicksal deutet.

\section{Anerkennung, Verdinglichung und Skepsis}

Nach dem Durchgang durch Cavells Shakespeare-Interpretationen sollen nun in einem letzten Schritt einige Schlussfolgerungen gezogen werden, die erhellen, inwieweit diese Interpretationen Aussagen über das Verständnis menschlicher Emotionen ermöglichen. Dabei kann mit einem sehr allgemeinen Punkt angefangen werden: In der Perspektive Cavells dienen die thematisierten Emotionen offensichtlich dazu, den Charakteren Skripte zu liefern, die sie in die Lage versetzen, Wissen zu verdrängen oder nicht anzuerkennen. Sie tun das auf unterschiedliche Weise, indem sie etwa wie in der Scham Bedingungen schaffen, unter denen die mit dem eigenen Wissen verbundenen praktischen Implikationen nicht umgesetzt werden können, oder wie in der Eifersucht nach Kriterien für Treue und Verlässlichkeit verlangen, die unmöglich beweisen können, was sie doch beweisen sollen, aber sie tun es in allen Fällen, indem die Stimme des anderen unhörbar wird.

Das Bild für diese Unhörbarkeit, das Cavell vor allem in seiner Interpretation des Wintermärchens heranzieht, ist das der Versteinerung des anderen, und das heißt: seiner Verdinglichung. Genau in diesem verdinglichenden Zug liegt die Nähe dieser Emotionen zum philosophischen Skeptizismus. Es sind in diesem Zusammenhang vor allem drei Aspekte des Skeptizismus, die für die Analyse der Emotionen relevant werden. Sie bündeln sich in der Beschreibung der typischen Situation des klassischen Skeptizismus (Cavell denkt vor allem an die Position des zweifelnden Ichs in den Meditationen Descartes'). Dieser Skeptizismus stellt sich die typische skeptische Situation als eine der Begegnung von Individuum und Welt vor, nicht aber als eine von Individuum und Individuum. Der Zweifel der klassischen Skepsis offenbart sich in Fragen nach der Erkennbarkeit der Welt oder einzelner Gegenstände in ihr, nicht aber in Fragen nach der Erkennbarkeit des anderen. Für Cavell heißt das: Der klassische Skeptiker ist in der Regel allein (vor dem Kamin), er befragt Gegenstände (ein Stück Wachs) und er sucht nach einer epistemisch bestimmten Gewissheit. Was die hier thematisierten Emotionen von dieser Beschreibung unterscheidet, ist, dass sie zunächst explizit in intersubjektiven Kontexten zur Geltung kommen, sich dann aber von diesen entfernen oder sie gar zerstören. Am Ende sind die Protagonisten allein, sie haben die konkreten anderen verdinglicht (getötet), und sie haben versucht, ihre Zweifel mithilfe kognitiver Anstrengungen zu überwinden (und sind genau dadurch in die Katastrophe gelaufen). Sie korrigieren den erkenntnistheoretischen Skeptizismus damit in dem Maße, in dem sie weniger „kognitiv“ sind und den Gegenstand des Zweifels gleichsam beleben, sodass am Ausgangspunkt der Skepsis die Geltungsansprüche (Aussagen) anderer stehen, an denen wir zweifeln können oder auch nicht, aber sie partizipieren auch an ihm, indem sie sich zunehmend von diesen intersubjektiven Kontexten entfernen und damit Zustände schaffen, in denen andere 
nicht länger Geltungsansprüche, Aussagen oder Behauptungen vorbringen können, weil sie tot oder versteinert sind oder schlicht der (eigenen und ihrer fremden) Gegenwärtigkeit verlustig gehen. ${ }^{37}$ Emotionen wie Scham, Eifersucht oder Ekel liefern im Kontext dieser Narrative die Motive und die Motivation, die zu dieser Enteignung und Auslöschung des Du führen und am Ende eine Welt zurücklassen, die nicht mehr spricht und folglich nicht mehr gehört wird. Sie erkennen damit die Bedürftigkeit des Subjekts und seine Angewiesenheit auf die Unterstützung anderer nicht an, sie leugnen sie vielmehr und fungieren damit als Instrumente der Nicht-Anerkennung und Missachtung der anderen.

Es mag an diesem Punkt hilfreich sein, den Cavellschen Anerkennungsbegriff gegen mögliche Missverständnisse in Schutz zu nehmen. Das ist auch deswegen nötig, weil Cavell selbst das Scheitern solcher Figuren wie Lear, Leontes oder Othello immer wieder mit ihrem Unvermögen in Verbindung bringt, den anderen oder die Welt anzuerkennen. Für Cavell dient beispielsweise Leontes als Porträt eines Skeptikers, dem sich die Welt entzieht. Auf diesem Porträt lässt sich das „Scheitern des Wissens“ als Scheitern der Anerkennung verfolgen, „was unter anderem bedeutet, dass das Ergebnis des Scheiterns keine Unwissenheit, sondern ein Nicht-wissen-Wollen ist, kein bestreitbarer Zweifel, sondern ein unversöhnliches Leugnen, eine willentliche Unwissenheit, die einer Auslöschung gleichkommt " ${ }^{38}$ Wenn das Scheitern der Anerkennung also als Leugnen eines Wissens gedeutet wird, was ist dann Anerkennung? Wie Cavells Beschreibung gescheiterter Anerkennung schon deutlich macht, wäre es an diesem Punkt falsch, den Begriff der Anerkennung als Gegenbegriff zum Wissen oder als Voraussetzung des Wissens einzuführen. Diesen Zug macht Axel Honneth, wenn er unter anderem mit Blick auf Cavell das Phänomen der „Anerkennungsvergessenheit“ als einen Prozess beschreibt, „durch den in unserem Wissen um andere Menschen und im Erkennen von ihnen das Bewußtsein verlorengeht, in welchem Maß sich beides ihrer vorgängigen Anteilnahme und Anerkennung verdankt“ ${ }^{39}$ Auch wenn nicht ganz klar ist, wie Honneth in dieser Passage den Begriff ,Vorrang“ verstanden wissen will, lässt sich nicht ausschließen, dass er die anerkennende Haltung als Alternative oder gar als normative Grundlage der erkennenden oder wissenden Haltung bestimmt. Zwar deutet auch Cavell in seinem Aufsatz Knowing and Acknowledging darauf hin, dass etwa mein Unvermögen, mit Anteilnahme auf den Schmerz des anderen zu reagieren, implizieren kann, dass ich nicht weiß, dass er Schmerzen hat, aber nichts deutet darauf hin, dass eine Form der anerkennenden Anteilnahme grundsätzlich Voraussetzung des Erkennens oder Wissens ist. ${ }^{40}$ Wenn ich nicht weiß, dass jemand Schmerzen hat, bin ich nicht in der Lage, diesen Schmerz anzuerkennen, das heißt, ich reagiere nicht, hole keinen Arzt, tröste nicht, besorge keine Medizin; weiß ich es andererseits, heißt das nicht, dass ich mit Anteilnahme reagiere. Ohnehin wäre es missverständlich, Cavells Begriff der Anerkennung zu eng mit dem Begriff der Reaktion zu verknüpfen, und noch missverständlicher, ihn mit dem Begriff der positiven, anteilnehmenden Reaktion zu verknüpfen. Reagiere ich gleichgültig oder mit Hohn und Spott auf den Schmerz des anderen, dann erkenne ich diesen Schmerz an, da ich auf den Schmerz des anderen reagiere, den ich so oder so zur Kenntnis genommen habe. In meinen Reaktionen ist der andere durchaus gegenwärtig, auch wenn ich ihm nicht helfe oder mich sogar aktiv von ihm abwende. Selbst wenn ich den Schmerz des anderen wahrnehme und überhaupt nicht reagiere, handelt es sich um eine Form der Anerkennung. ${ }^{41}$ Ich tue zwar nicht, was in der Situation angemessen ist, ich tue auch nicht, was der andere von mir mit seinem Schmerzschrei vermutlich fordert, aber ich bin auch nicht im strengen Sinne taub oder blind. Ich habe den Schmerz wahrgenommen und mich entschieden, so oder so zu reagieren oder gar nicht zu reagieren. In die- 
sem Sinne fällt der Begriff der Anerkennung für Cavell nicht mit spezifischen positiven oder negativen Reaktionsweisen zusammen: „Es handelt sich bei ihm [dem Anerkennungsbegriff] nicht um die Beschreibung einer gegebenen Reaktion, sondern um eine Kategorie, mit deren Hilfe eine gegebene Reaktion bewertet wird. “42 Wir können ein Verhalten nur in dem Maße als rücksichtslos, gleichgültig, grob, missachtend oder freundlich bezeichnen, in dem wir im anderen ein Wesen erkennen, dass Forderungen an uns richtet, die einen normativen Gehalt haben. Was folglich vorgängig ist in unseren Interaktionen mit anderen Subjekten, sind ihre Forderungen und Ansprüche [claims], auf die wir immer schon reagieren oder nicht reagieren. Entsprechend schwer fällt es, im Kontext der Cavellschen Überlegungen einen Gegenbegriff zum Begriff der Anerkennung zu formulieren. Letztlich ist es der Wittgensteinsche Begriff der „Seelenblindheit“, der hier noch am besten einfängt, was es heißt, einen Menschen nicht als Menschen zu behandeln, und das heißt jetzt: nicht anzuerkennen. Lear, Othello, Leontes und Coriolanus scheitern in ihrer Anerkennung des anderen nicht schon in dem Augenblick, in dem sie mit Wut oder Eifersucht reagieren; vielmehr scheitern sie in dem Augenblick, in dem diese Reaktionen dazu führen, für die Seele des anderen blind zu werden und damit den anderen (und sich selbst) zu verdinglichen (zu versteinern, zu theatralisieren). Wollte man es noch dramatischer sagen, dann könnte man es auch so formulieren: Der eigentliche Gegenbegriff zum Begriff der Anerkennung ist der Begriff des Todes als radikale Form der Nichtpräsenz. ${ }^{43}$

Aus diesen Überlegungen folgt nun auch, dass Anerkennung und Wissen sich nicht wie Gegenbegriffe verhalten, sondern auf komplexe Weise miteinander verzahnt sein können. ${ }^{44}$ Es zeichnet die Tragödien Shakespeares aus, dass in ihnen Anerkennung scheitert, weil ein Wissen geleugnet wird oder weil die Art und Weise des Umgangs mit dem Wissen den Forderungen des anderen nicht gerecht wird. Mehr noch, da dieses Leugnen willentlich geschieht, können die Charaktere auch nicht das Schicksal anrufen, um ihr Leiden mit Sinn zu füllen. Sie selbst sind es, die sich den anderen (und sich selbst) entziehen. Sie unterstehen damit auch nicht der ,alltagsweltlichen Sogwirkung des erkenntnistheoretischen Modells“, sondern der alltagsweltlichen Skepsis und ihrer Sogwirkung selbst. ${ }^{45}$ In Lear, Othello, Leontes und Coriolanus Porträts des philosophischen Skeptikers zu sehen heißt nicht, in ihnen philosophische Skeptiker zu sehen. Es heißt, die Alltäglichkeit der Skepsis anzuerkennen.

Aus diesen Überlegungen folgt schließlich auch, dass der Begriff der Anerkennung konstitutiv mit bestimmten Selbstverständnissen verbunden ist. Blind für die Forderungen anderer zu sein heißt, sich ihnen zu verschließen, sich nicht öffnen, sich nicht präsent machen. In diesem Sinne geht die gescheiterte Anerkennung des anderen einher mit einer gescheiterten Anerkennung des eigenen Selbst durch sich selbst und durch andere. Folglich muss die Analyse des Scheiterns der Anerkennung mit einer Analyse der unterschiedlichen Selbstverhältnisse einhergehen, die zum Scheitern intersubjektiver Anerkennung führen und, wie hinzugefügt werden kann, mit einer Analyse der sozialen und kulturellen Bedingungen, die unweigerlich in allen Formen des Selbstverhältnisses präsent sind.

\section{Narrative als Methode des Verstehens menschlicher Emotionen}

Nach diesen Überlegungen zur Anerkennung soll nun die Thematik der Emotionen abschließend aufgegriffen werden. Welche Einsichten lassen sich aus den literarischen Fallbeispielen Cavells ziehen? Es sind vor allem zwei Aspekte, die hier erwähnt werden müssen. Zum einen 
zeigen Cavells Interpretationen, dass das Wirken einzelner Emotionen nur dann angemessen verstanden werden kann, wenn es auf ein Selbstverständnis bezogen wird, das einen holistischen Charakter hat und nicht auf einzelne Überzeugungen, Handlungen oder Reaktionen reduziert werden kann. Nur ein Individuum, das die eigene Reinheit in der Reinheit des Partners widergespiegelt sehen will, wird in der Weise Othellos auf einen vermuteten Treuebruch reagieren. Und nur ein Individuum, das die Separatheit des anderen (die sich etwa in seiner eigenständigen Sexualität ausdrückt) fürchtet, wird die Eifersucht einsetzen, um dieses Faktum zu leugnen. Dieser Punkt scheint angesichts der vorliegenden Beispiele evident zu sein, aber er muss dennoch hervorgehoben werden, da direkt repräsentationalistische Ansätze die Neigung haben, Emotionen isoliert zu betrachten, und ihre Einbettung in Selbstverständnisse nicht thematisieren. Sie tun dies, weil sie, so lässt sich vermuten, den Subjektivierungsschub fürchten, den eine stärkere Berücksichtigung individueller Selbstverständnisse mit sich bringt. Ein Grund, weswegen hier Cavells Interpretationen beispielhaft herangezogen werden, hängt mit der Tatsache zusammen, dass sie den Zugang zum Phänomen menschlicher Emotionen zunächst tatsächlich subjektivieren, indem sie die emotionalen Reaktionen der beschriebenen Figuren an ihre je unverwechselbare Identität und ihr eigentümliches Selbstverständnis binden. Ohne Bezug auf dieses Selbstverständnis können die Emotionen, um die es geht, gar nicht angemessen verstanden oder erklärt werden. Selbstverständnisse umfassen dabei die Art und Weise, in der Subjekte sich sehen und beschreiben, die Art und Weise, in der sie sich und ihr Handeln deuten. Welche Relevanz eine Annahme besitzt, welches Gewicht eine Überzeugung gewinnt, welche Handlungsfolgen Scham, Eifersucht oder Ekel nach sich ziehen - all das sind Fragen, die nur mit Verweis auf narrativ zu explizierende Selbstverständnisse beantwortet werden können. Entsprechend sinnlos ist es, einzelne Emotionen mit spezifischen Überzeugungen in Verbindung zu bringen, wie manche klassischen Kognitivisten das glaubten tun zu können. Ob eine Überzeugung, eine Handlung oder eine Reaktion als eifersüchtig gekennzeichnet werden kann, hängt nicht an ihr selbst, sondern an ihrer Einbettung in ein Geflecht von Überzeugungen, Handlungen und Reaktionen, das sinnvoll als Geflecht der Eifersucht beschrieben werden kann.

Damit dieses Geflecht überhaupt als eines der Eifersucht entziffert wird, muss es sicherlich Elemente aufweisen, die typisch für Eifersucht sind. Othello reagiert eifersüchtig auf Desdemona und Casio, weil er unter dem Einfluss Jagos befürchtet, Desdemona zu verlieren; Leontes reagiert eifersüchtig auf Hermione und Polixenes, weil er vermutet, dass sie eine Liebesbeziehung führen. Prozesse des Verstehens sind stets eingelassen in Vorverständnisse, die gleichsam die Richtung vorgeben, die der Deutungsprozess einschlägt. In diese typische Grundkonstellation hinein aber konstruiert Cavell nun eine andere Eifersucht, von der die typische Eifersucht gleichsam ablenkt. Diese andere Eifersucht gilt weniger dem konkret befürchteten Verlust des anderen als dem Faktum seiner Separatheit, das die konkrete Trennung überhaupt erst möglich macht. Cavell lässt keinen Zweifel daran, dass diese Eifersucht, die der Existenz des anderen gilt und die mit absoluten Besitzansprüchen verbunden ist, den Begriff der Eifersucht aus seiner normalen Grammatik herausdreht. Wie noch deutlich werden wird, ist das Ungewöhnliche aber nicht das Andere des Gewöhnlichen, sondern dessen unabdingbare Kehrseite. Folgt man seiner Deutung in ihrer ganzen Exzentrizität, wird zumindest klar, dass die typische Grammatik der Eifersucht nicht das letzte Worte hat, wenn es darum geht, die Verhaltensweise dieser Figuren zu verstehen. Sie verhalten sich gewissermaßen noch einmal zu ihrer konkreten Eifersucht, setzen sie ein, geben ihr im Lichte ihres Selbstverständnisses eine eigentümliche Wendung. So wird aus der Furcht, den anderen zu verlieren, der unmenschliche (und 
doch menschliche) Wunsch, ihn nicht verlieren zu können. Ist das wahnsinnig? Ist es irrational? Es ist wahnsinnig, denn Lear, Othello und Leontes sind, in unterschiedlichen Phasen, wahnsinnig und töten (verbannen und verdammen), was sie vorgeblich nicht verlieren wollen. Ist es ungewöhnlich? Ein Blick in die Zeitung genügt, um diese Frage ihrer nahe liegenden Antwort zu berauben. Wenn verlassene Väter ihre Frauen töten und verlassene Mütter ihre Kinder töten, sagen die Nachbarn in der Regel: Sie waren so normal. Wir neigen dazu, das nicht ernst zu nehmen, weil es unheimlich ist.

Der Ausgang vom Selbstverständnis muss nicht zu atomistischen Konsequenzen führen. ${ }^{46}$ Selbstverständnisse bestehen aus Deutungen, in die soziale Erwartungen und kulturelle Einflüsse hineinspielen, ein Punkt, den Cavell nicht hinreichend betont, weil er zu einer Anthropologisierung der Strukturen des Zweifels neigt. ${ }^{47}$ Der Versuch, ein Selbstverständnis vollständig zu verstehen, kann erfordern, es in einen größeren soziokulturellen Kontext einzubetten, sodass die Philosophie in ihrer Deutungskompetenz schnell an ihre Grenzen stößt. Der Ausgang vom Selbstverständnis impliziert darüber hinaus keine Selbsttransparenz des fühlenden Subjekts. Selbstverständnisse leiten das Subjekt nicht nur dann, wenn es sich darüber im Klaren ist, wie es sich versteht. Sie bleiben eher im Hintergrund, fungieren als Rahmen, in den soziale, kulturelle und individuell-idiosynkratische Einflüsse hineinspielen. Dass die Emotionen das gegenläufige Wissen der Subjekte überleben können, dass sie also andauern, obwohl es keinen Anlass mehr für sie gäbe, lässt sich durch einen Verweis auf die Rolle von Selbstverständnissen erklären, die offensichtlich über verschiedene Schichten verfügen. Wenn das Wissen um den Unsinn der eigenen Eifersucht die Eifersucht nicht beseitigt, könnte es an unartikulierten Vermutungen und Verdächtigungen liegen, die sich dem bewussten Subjekt entziehen. Es könnte aber auch daran liegen, und das ist der Fall bei den von Cavell herangezogenen Figuren, dass dieses Wissen abgelehnt wird, weil es das verhindert, was mit Blick auf Leontes als Rache am Leben bezeichnet wird. Dieser Wunsch, der sich der Emotion der Eifersucht bedient, um praktisch wirksam zu werden, widerspricht nicht dem eigenen Wissen, er versucht vielmehr, es als solches zu verdrängen, weil diese Figuren sich, wenn man so will, nicht mit diesem Wissen identifizieren.

Wird die Verwobenheit einzelner Emotionen mit Selbstverständnissen ernst genommen, verschiebt sich die Art, in der Emotionen als angemessen oder unangemessen bezeichnet werden, und zwar in normativer und methodischer Hinsicht. Der direkte Repräsentationalismus glaubt, diese Frage durch einen Vergleich der in der Emotion enthaltenen Weltverwiesenheit mit der Welt an sich beantworten zu können. So wie wir Wahrnehmungen daraufhin überprüfen können, ob sie wirklich unverzerrt sind, so können wir in diesem Modell Emotionen an der Realität abgleichen, und zwar selbst dann, wenn es überhaupt erst die Emotionen sind, die uns die Welt evaluativ erschließen. Ob meine Empörung über dich berechtigt ist, ob sie angemessen ist, weiß ich erst dann, wenn ich prüfe, ob du mich wirklich verletzt hast. Ich muss meine Aufmerksamkeit nach außen richten, muss meine Emotion mit den Fakten abgleichen, und erst dann weiß ich, ob sie angemessen ist oder nicht. Gemessen an diesem Kriterium der Angemessenheit sind alle von Cavell in seinen Shakespeare-Interpretation thematisierten Emotionen naturgemäß unangemessen. Lear weiß, dass Cordelia ihn liebt, Othello weiß, dass Desdemona nicht wirklich untreu ist, Leontes weiß, dass sein Kind sein Kind ist, und Coriolanus weiß, so steht zu vermuten, dass er kein Gott ist. Alle diese Figuren wissen, was ihre Emotionen doch leugnen. Folglich sind ihre Emotionen unangemessen. Die Wirklichkeit sieht schlicht anders aus. 
Es ist zweifellos richtig, auf die Unangemessenheit dieser Emotionen hinzuweisen. Gleichwohl stellen sich einige Fragen, die die Basis dieses Urteils betreffen. So ist zum einen das Wissen, um das es sich in allen diesen Fällen handelt, ein instabiles Wissen, weil es sich bereits als solches aus einer Praxis gelöst hat, in deren Rahmen der Zugang zum anderen kein primär epistemologischer ist. Dass Leontes weiß, dass sein Sohn sein Sohn ist, oder den Versuch unternimmt, ein solches Wissen durch Nachfrage zu etablieren, impliziert bereits eine Entfernung im praktischen Verhältnis, impliziert einen Gegenwärtigkeitsverlust, der durch Wissen nicht kompensiert werden kann oder im Wissen und Wissenwollen überhaupt erst auf unerkannte Weise artikuliert wird. ${ }^{48}$ Die Emotion widerspricht in diesem Sinne nicht dem, was gewusst wird, sie ist vielmehr die Kehrseite der Unsicherheit des Wissens. Ist das aber der Fall, wird es schwierig oder sogar irrig, ihre Beurteilungsbasis in dem aufgehen zu lassen, was (etwa durch genaues Hinschauen) gewusst werden kann. Wenn es nicht entscheidend ist, etwas über den anderen oder über sich selbst zu wissen (über seine Liebe, sein Sohnsein, über die eigene Zugehörigkeit zu den anderen als Sprachwesen), sondern etwas an ihm und an sich selbst anzuerkennen (seine Liebe, sein Sohnsein, die eigene Zugehörigkeit zu den anderen als Sprachwesen), wobei Anerkennung eine voraussetzungsreiche Form der wechselseitigen Gegenwärtigkeit meint, dann verschiebt sich die Beurteilungsbasis der Emotion, weil ihre Angemessenheit oder Unangemessenheit nicht länger an ihrer repräsentationalen Übereinstimmung mit Wirklichkeit gemessen wird, sondern an ihrem Vermögen, in praktisch folgenreicher Weise Strukturen der Anerkennung zu ermöglichen oder zu artikulieren, die sich nicht durch das erfassen lassen, was wahrgenommen werden kann, weil das Faktum der Wahrnehmung (oder des wahrnehmungsbasierten Wissens) schon als Form des Vermeidens der Ansprüche und Forderungen des anderen gedeutet werden kann. In dem Maße, in dem Scham, Eifersucht und Ekel in den Narrativen Cavells diese Anerkennungsstrukturen zerstören, sind diese Emotionen unangemessen.

Die Basis der Beurteilung einzelner Emotionen verändert sich damit, da diese Emotionen nicht länger isoliert betrachtet werden können, sondern einen konstitutiv intersubjektiven Gehalt bekommen, dessen konkrete Form je situativ bestimmt werden muss. Gegenwärtigkeit als Basis der Anerkennung umschreibt dann das Vermögen, für das je situativ Geforderte und Beanspruchte sensibel zu sein, ohne dabei behaupten zu müssen, dass diese Forderungen und Ansprüche sinnlich wahrgenommen werden. Die Kategorie der Anerkennung ist an diesem Punkt abstrakt genug, um nicht vorentscheiden zu müssen, auf welche Weise Forderungen und Ansprüche aufgenommen werden. Emotionen (wie Anteilnahme) können situative Formen der Sensibilität ermöglichen, sie können aber auch die eigene Wirklichkeitswahrnehmung durchdringen und verzerren. Sie tun dies jeweils als Bestandteil eines Selbstverständnisses, zu dem auch der Wunsch gehören kann, die eigenen Emotionen an Wirklichkeit zu orientieren, weil nur dadurch missliche Folgen der eigenen (und fremden) Emotionalität verhindert werden können. Ein Vorteil, der darin besteht, das Selbstverständnis als wesentlichen Kern der Emotionsdeutung heranzuziehen, hat gerade mit der Tatsache zu tun, dass Selbstbilder nicht beanspruchen, Wirklichkeit abzubilden oder zu repräsentieren. In ihnen bündeln sich Antworten auf die Frage danach, wer man - auch im Verhältnis zu anderen - sein will, und diese ethische Frage ist eine andere Frage als die nach dem Verhältnis der eigenen Emotion zur Wirklichkeit. Emotionen sind im Rahmen eines je narrativ zu entfaltenden Selbstverständnisses unangemessen, nicht aber an sich. In ihre Beurteilung fließt damit tatsächlich etwas ein, was über sie hinaus geht, aber das ist nicht ihr Bezug zu einer außeremotionalen Realität, 
sondern ihr Bezug auf ein Selbstverständnis in seinem Verhältnis zu anderen Selbstverständnissen. So lässt sich auch der Wunsch deuten, eine praktisch relevante Frage (Bist du mein Sohn?) durch Befragung der eigenen Wahrnehmung zu klären. Dieser Wunsch verrät, dass das Hinzuziehen der eigenen Wahrnehmung kein neutraler Akt im Interesse der Wahrheitsfindung ist, sondern selbst wiederum einem emotional geleiteten Wunsch entspringen kann, der in seiner Irrationalität durch den Verweis auf die scheinbar neutrale epistemische Instanz der Wahrnehmung gerade verborgen bleiben soll. ${ }^{49}$ Warum befragt Leontes seinen Sohn? Weil er eifersüchtig ist. Warum ist er eifersüchtig? Darum geht es, unter anderem, in dem Stück Das Wintermärchen.

Was die Figuren Shakespeares in der Deutung Cavells interessant macht, ist ein Zug, der durchaus typisch ist für viele Emotionen des Menschen und der im Modell des direkten Repräsentationalismus nicht erfasst wird. Wenn unsere Wahrnehmungen emotionsgeleitet sind, schärfen sie unseren Blick für Situationen, in denen die Emotion sich gewissermaßen bestätigt sieht. Dass der eifersüchtige Leontes überall Hinweise sieht, die seiner Eifersucht Recht geben, impliziert nicht zwangsläufig, dass er die Wirklichkeit verzerrt wahrnimmt. Manche Inszenierungen des Wintermärchens spielen an diesem Punkt mit der Ambivalenz der Situation, indem sie Hermione tatsächlich in großer Vertrautheit mit Polixenes zeigen. Die Frage, die sich hier ergibt, liegt auf der Hand: Wann ist die Wirklichkeit so, dass die Eifersucht absolut berechtigt ist? Muss ein Kuss beobachtet werden? Reicht ein Streichen über die Hand, ein verstecktes Lächeln? Was die Wahrnehmungsanalogie zu wenig berücksichtigt, ist die Interpretationsabhängigkeit der Eifersucht selbst, für die es keine eindeutigen evaluativen Eigenschaften an den Dingen gibt, die sie letztgültig als berechtigt ausweisen könnten. Genau das ist einer der Gründe für die Willkür der Eifersucht und, wie man hinzufügen kann, für die Willkür vieler komplexer Emotionen. Sie machen sich die Abwesenheit eindeutiger Kriterien für das Zuschreiben von Zuständen (Untreue, Sohnsein und so weiter) zu Nutze, indem sie diese Uneindeutigkeit mit ihrer eigenen Eindeutigkeit ausfüllen. Sie verzerren folglich nicht eine Wirklichkeit, die eindeutig wäre, sie verzerren vielmehr eine Wirklichkeit, die uneindeutig ist, und isolieren deswegen ihre Subjekte. Weil unsere gewöhnlichen Praktiken kein festes Fundament jenseits der zumeist implizit vorausgesetzten praxisrelevanten und praxisbestimmenden Kriterien haben, können Emotionen in bestimmten Narrativen ihr skeptisches Gift entfalten. Willkür darf folglich nicht heißen: unberechtigt oder unangemessen. Dass Emotionen eine welterschließende Seite haben, heißt, dass sie konstitutiv für den Zustand sind, der durch sie hindurch erschlossen wird. Das ist der Grund, weswegen die subjektive Perspektive zum Verständnis der Emotion unabdingbar ist. Die richtige Antwort auf Leontes (oder Othello) lautet entsprechend nicht: ,Sieh' genau hin, da ist nichts! ', denn für Leontes ist etwas da. Dass nichts da ist, müsste gewissermaßen erst einmal geklärt werden und kann nicht einfach wahrgenommen werden (wir müssten klären, welche Kriterien der Eifersucht für uns Geltung haben; Leontes hat diese Kriterien gleichsam in Frage gestellt oder glaubt, sich sogar auf sie berufen zu können). In extremen Fällen sucht der Paranoide Situationen, in denen er berechtigt ist, seine Emotionen zu empfinden. Wer überall Betrug wittert, hat ein feines Gespür für Situationen, in denen er betrogen wird. Seine Emotion wäre in diesen Situationen nach Auskunft des direkten Repräsentationalismus vollkommen berechtigt. Und doch stimmt etwas nicht an ihr, und das drücken wir aus, wenn wir von Paranoia sprechen. Aber was stimmt nicht? Die Antwort darauf kann nicht eindeutig ausfallen. Wir halten ein Leben, das überall Verrat wittert, für ein unglückliches Leben, das viel 
vermeidbares Leiden beinhaltet. Das könnte ein Grund dafür sein, die Emotionen des Paranoiden für unangemessen zu halten.

Narrative, so hieß es, sind methodische Instrumente der Interpretation menschlicher Emotionen im Kontext eines Selbstverständnisses. Sie sind damit der Reflex der konstitutiven Rolle, die Subjektivität im Prozess des Verstehens menschlicher Emotionalität spielt, und tragen in diesem Sinne zur Individualisierung der Emotion bei. Dass Emotionen aber überhaupt dazu einladen, narrativ rekonstruiert zu werden, hängt an der ihnen eigenen Historizität, durch die wir in die Lage geraten zu erläutern, wie sich eine Emotion im zeitlichen Verlauf entwickelt hat. Einen solchen zeitlichen Verlauf besitzen nicht alle mentalen Phänomene. So können wir nicht für alle unsere Wünsche einen solchen Verlauf angeben. Manche unserer Wünsche entstehen und vergehen spontan, ohne dass wir Auskunft darüber geben können, warum sie entstanden sind. Vielleicht gehen Wünschen (wie Emotionen) neurophysiologisch beschreibbare Prozesse im Körper voraus, sodass sie so etwas wie eine zeitliche Spur besitzen, aber diese Spur kann uns nur Aussagen über die Kausalität der Wunschentstehung liefern, nicht aber über interpretatorisch zu erschließende Gründe für das Haben von Wünschen. Emotionen dagegen lassen sich in ihrem Entstehen (und Vergehen) häufig auf Ursprungsbedingungen zurückführen, zu denen etwa das Vorliegen bestimmter Wünsche oder die Reaktion auf affektive Zustände gehört. ${ }^{50}$

Dass Emotionen in diesem Sinne über Historizität verfügen, ist eine der Vorbedingungen für ihre Narrativität, aber es spricht nichts dafür, Historizität mit Narrativität gleichzusetzen, was etwa geschieht, wenn im Kontext narrativer Emotionstheorien von einem „unmittelbar narrativen Erleben" der Emotion gesprochen wird. ${ }^{51}$ Gemeint ist damit, dass Emotionen an sich schon über eine narrative Struktur verfügen, wobei der Begriff des Narrativen hier eine synthetisierende Kraft meint, die aus den pluralen Komponenten der Emotion - aus ihren phänomenalen, expressiven, behavioralen und körperlichen Zügen - eine einheitliche Kategorie des Erlebens macht. Wird der Begriff der Narration dagegen eindeutig als methodisches Instrument der Interpretation von Emotionen behandelt, ist es sinnlos, Emotionen an sich als narrativ strukturiert zu beschreiben. Wenn sie als unmittelbar empfundener Zustand auftreten (ein Anfall von Hass, von Scham, von Eifersucht), mag die Annahme berechtigt sein, wonach dieser Zustand vom Subjekt einheitlich empfunden wird, dieses Einheitserleben jedoch verdankt sich keiner narrativen Leistung der Emotionen selbst. Narrativ werden Emotionen vielmehr erst in dem Augenblick, in dem wir uns als Subjekte der Emotion oder in der Beobachterperspektive darum bemühen, ihnen einen Sinn abzugewinnen, um sie zu verstehen. Narrative sind in diesen Sinne Ergebnisse einer interpretatorischen Konstruktionsleistung der Subjekte. ${ }^{52}$ Damit ist angedeutet, dass wir auch dann Emotionen haben, wenn wir sie nicht erzählen oder nicht versuchen, sie zu verstehen. Und es ist angedeutet, dass Emotionen gegenüber der Narration eine gewisse Autonomie behalten, die etwa dann zum Tragen kommt, wenn sie - wie im Falle Lears - in das Vermögen der verstehenden Rekonstruktion eingreifen oder es sogar zerstören. Noch für den scheinbar affektlosen wissenschaftlichen Beobachtungsblick gilt, dass er auf der Basis eines „Gefühlskollektivs“ möglich wird. „Die Lehrzeit in einer Wissenschaft“, so Lorraine Daston, ,initiiert den Neuling in Fühlweisen, genauso wie in Seh-, Verstehens- und Handlungsweisen. "53 Selbst wenn wir also ein wissenschaftliches Narrativ anfertigen, um einzelne oder kollektive Emotionen zu verstehen, steht das Vermögen der Narration selbst unter dem Einfluss von Emotionen, die Teil eines wissenschaftsgeschichtlichen Narrativs werden können, selbst aber in diesem nicht aufgehen. Das heißt 
schließlich auch, dass die Rede vom Narrativ nicht zu stark an literarische Narrative angebunden werden sollte. Literarische Narrative haben sicherlich häufig einen exemplarischen Charakter, wenn es darum geht, die Grundlagen und den Verlauf emotionstypischer narrativer Strukturen zu veranschaulichen, weswegen Autoren wie Nussbaum die Auffassung vertreten, unser Emotionsverständnis sei in hohem Maße von literarischen Narrativen geprägt, aber ihnen kommt nicht per se eine größere Explikationskraft zu als nicht-literarischen Narrativen. Es seien hier nur zwei spezifische Aspekte literarischer Narrative erwähnt: Auf Grund ihrer individualisierenden und partikularisierenden Züge gelingt es ihnen besser, das Verständnis emotionaler Phänomene auf die besonderen Umstände zu lenken, unter denen Emotionen entstehen und vergehen. Auch wenn wir selbst als Rezipienten nicht davon ausgehen, dass die Emotionen Lears oder Othellos unsere Emotionen in gleicher Lage wären, verstehen wir auf der Basis der Narrative besser, was sie zu ihren Emotionen getrieben hat. Wir können folglich verstehen, wie in ihrer Lage Emotionen wie Scham oder Eifersucht entstehen konnten. Darüber hinaus, darauf ist verwiesen worden, erfassen wir anhand der literarischen Narrative besser, dass das Vermögen, sich selbst und andere zu erzählen, an Voraussetzungen gebunden ist, die zerstört werden können oder von Anfang an nicht vorhanden sind. Literarische Narrative können in diesem Sinne als solche Metanarrative sein, in denen die Bedingungen des Narrativen verhandelt werden. Sie erzählen das Erzählen.

Es gibt also eine Reihe von guten Gründen dafür, weswegen in wissenschaftlichen Emotionstheorien häufig Bezug auf literarische Narrative genommen wird. Aber diese Bezugnahme deutet nicht darauf hin, dass alle Emotionen nur in literarischen Narrativen entschlüsselt werden können, sondern darauf, dass ihr Verständnis allgemein narrative Strukturen hat. Unabhängig von literarischen Narrativen indizieren Narrative eine synthetisierende und eine individualisierende Seite. Wenn wir versuchen, eine konkrete Emotion zu verstehen, haben wir es mit einer Fülle von Daten zu tun, die wir unter Bezug auf historische, kulturelle, psychologische und soziologische Aspekte entschlüsseln können. Eine Aufgabe des Narrativs ist es, diese Datenfülle zu vereinheitlichen, sodass wir mit Berechtigung von Lears Scham oder Leontes' Eifersucht sprechen. Diese Vereinheitlichung individuiert die Emotion aber nicht im ontologischen, sondern nur im hermeneutischen Sinne. Sie ist darüber hinaus in der Lage, im Kontext wissenschaftlicher Narrative typische Merkmale individueller Emotionserfahrungen zu bestimmen, sodass der konstitutive Bezug auf Selbstverständnisse nicht zwangsläufig im schlechten Sinne subjektivistisch wird. Die Rede von „Gefühlskollektiven“ bezieht letztlich ihre Berechtigung aus der Zusammenschau einzelner Narrative, die als solche erlaubt, Parallelen und Ähnlichkeiten der emotionalen Reaktionsweise zu erkennen.

Der Begriff des Narrativen ist, so steht zu vermuten, damit noch immer nicht sehr klar von anderen Begriffen abgegrenzt. Wesentlich zum Verständnis des Phänomens ist der Begriff des Verstehenwollens: „Wenn wir einem bestimmten Geschehen Sinn abzugewinnen versuchen, geschieht es oft, daß wir eine Geschichte über eine Ereignisabfolge erzählen, die zu

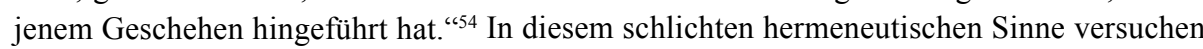
etwa die Neurowissenschaften nicht, das Phänomen der Emotionen zu verstehen, sie versuchen, es zu erklären. Mit anderen Worten, sie fragen nicht danach, was die Emotionen für die von ihnen betroffenen Subjekt bedeuten, welche Gründe sie für sie anführen und wie gut diese Gründe sind. Sie können diese Fragen nicht stellen, weil die Perspektive der Subjektivität für sie keine Rolle spielt. Nehmen wir, um ein letztes Beispiel zu nennen, zwei Menschen, die dankbar für eine Wohltat sind, die ihnen jemand zukommen lässt. Die Dankbarkeit 
des einen ist direkt und naiv, er freut sich über das, was er erhalten hat. Die des anderen vermischt sich mit Ressentiment gegenüber dem Wohltäter, weil die Gabe die eigene Bedürftigkeit schmerzhaft vor Augen führt. Hält man daran fest, dass hier zwei unterschiedliche Ausprägungen eines Phänomens vorliegen, das wir Dankbarkeit nennen, dann wird man die Unterschiede nur erfassen können, wenn die Vorgeschichten dieser Emotionen in den Blick kommen. Und in diese Vorgeschichte ist ein Verweis auf das Selbstverständnis der Individuen integriert. ${ }^{55}$ Es ist gerade die Notwendigkeit, auf das Selbstverständnis der Subjekte Bezug zu nehmen, die den Konstruktivismus narrativer Emotionstheorien gleichsam bindet. Dass wir überhaupt auf Narrative Bezug nehmen, wenn wir Emotionen verstehen wollen, hat mit dem thematisierten Ausgangspunkt im Selbstverständnis zu tun. Selbstverständnisse lassen sich in ihrer internen Komplexität oft nur narrativ erfassen, das heißt, sie erfordern von sich aus einen narrativen Zugang. Wenn wir wissen wollen, warum Lear mit Scham auf die Liebe Cordelias reagiert, müssen wir wissen, welche Rolle seine Machtlosigkeit, seine „Impotenz“ oder die Furcht vor Reziprozität für ihn spielen. Wir brauchen diese Verweise, weil wir nur so seine emotionale Reaktion plausibilisieren können.

Dennoch bleibt es am Ende dabei, dass die von Cavell verhandelten Emotionen der Skepsis einen eigentümlichen Zug behalten. Das kann auch nicht weiter überraschen, wenn man sich vor Augen führt, dass Cavell seine Deutung an Tragödien veranschaulicht. Die destruktiven Konsequenzen der Scham, der Eifersucht und des Ekels verweisen auf Selbstverständnisse, die gleichsam die Bedingungen positiver Emotionalität emotional leugnen. Wenn es zu dieser emotionalen Leugnung gehört, Wissen, das da ist oder leicht da sein könnte, nicht ernst zu nehmen oder in seiner ethischen Dimension falsch einzuschätzen (indem es überschätzt wird), dann offenbart sich an diesem Zug erneut die bisweilen unheimliche Autonomie der Emotionen, die es angesichts ihrer unlängst erfolgten philosophischen Rationalisierung hervorzuheben gilt. Kein Narrativ kann diesen Aspekt willkürlich wegdiskutieren.

Dr. Martin Hartmann, Johann Wolfgang Goethe-Universität, Institut für Philosophie, Grüneburgplatz 1, 60629 Frankfurt/M.

\section{Anmerkungen}

1 H. Steinfath, Emotionen, Werte und Moral, in: Die Moralität der Gefühle, hg. v. S. Döring u. V. Mayer, Berlin 2002, 105-122.

2 R. de Sousa, Emotional Truth, in: The Aristotelian Society, Ergänzungsband LXXVI (2002), 247-263, hier: 256.

3 J. McDowell, Non-Cognitivism and Rule-Following, in: ders., Mind, Value, and Reality, Cambridge/Mass. 1998, 198-218, hier: 200.

4 Ch. Taylor, Abschied von einer Theorie der indirekten Erkenntnis, in: Die Öffentlichkeit der Vernunft und die Vernunft der Öffentlichkeit. Festschrift für Jürgen Habermas, hg. v. L. Wingert u. K. Günther, Frankfurt/M. 2001, 53-76.

5 B. Helm, Emotions and Practical Reason: Rethinking Evaluation and Motivation, in: Noûs, 35:2 (2001), 190213, hier: 196 (Hervorhebung von Helm).

6 W. Shakespeare, King Lear/König Lear, Stuttgart 1973 (alle Zitate nach dieser Ausgabe).

7 Die bestätigende Anerkennung vollzieht sich dabei zunächst nur auf der Ebene von Sprechakten und nicht, wie etwa von Honneth gefordert, auf der Ebene von „Handlungen, Gesten und Mimik“ (vgl. A. Honneth, Unsichtbarkeit. Über die moralische Epistemologie von ,Anerkennung', in: ders., Unsichtbarkeit. Stationen 
einer Theorie der Intersubjektivität, Frankfurt/M. 2003, 10-27, hier: 15). Der Status von expliziten Sprechakten bleibt bei Honneth insgesamt eher undeutlich. Wenn es später heißt, Handlungen, Gesten und Mimik leisteten ihre Anerkennungsarbeit ,in Ersetzung, in Ergänzung oder in Unabhängigkeit von Sprechakten“ (19), wird immerhin angedeutet, dass explizite Sprechakte auch eine anerkennende Dimension besitzen können.

8 St. Cavell, The Avoidance of Love. A Reading of King Lear, in: ders., Disowning Knowledge in Seven Plays of Shakespeare, Cambridge 2003, 39-123, hier: 45.

9 Ebd., 49.

10 Ebd., $61 \mathrm{f}$.

11 Ebd., 58.

12 Ebd., 69.

13 Ebd., 120.

14 Ebd., 96: „Lear abdicates sanity for the usual reason: It is his way not to know what he knows, or to know only what he knows."

15 Cavell betont an vielen Punkten von The Avoidance of Love die Komplizenschaft zwischen Figuren und Zuschauern. Indem wir nicht sehen, was auch die Figuren nicht sehen, partizipieren wir gleichsam an ihrem Scheitern und sind so in die Tragödie ,impliziert“ (85). Auch wenn diese These viele fruchtbare Gedanken hervorbringt, untertreibt sie das mögliche Maß an ästhetischer Distanz und Differenz zwischen Figur und Zuschauer (vgl. J. Rebentisch, Ästhetik der Installation, Frankfurt/M. 2003, 25 ff.).

16 Vgl. A. Caverero, The Desire for One's Story, in: dies., Relating Narratives. Storytelling and Selfhood, London 2000, 32-45, hier: 33: „Precisely because it [the human existent] is exposable, it is narratable.“

17 Spinoza, Die Ethik, Stuttgart 1977, 329 u. 331 (Dritter Teil, Lehrsatz 35 und Anmerkung).

18 Vgl. J. Neu, Jealous Thoughts, in: Explaining Emotions, hg. v. A. O. Rorty, Berkeley 1980, 425-463.

19 St. Cavell, Othello and the Stake of the Other, in: ders., Disowning Knowledge, a. a. O., 125-142, hier: 133 (dt.: ders., Der Anspruch der Vernunft. Wittgenstein, Skeptizismus, Moral und Tragödie, Frankfurt/M. 2006, 773).

20 Ebd., 138 (dt.: Der Anspruch der Vernunft, a. a. O., 780).

21 Ebd., 130 (dt.: Der Anspruch der Vernunft, a. a. O., 768).

22 Ebd., 135 (dt.: Der Anspruch der Vernunft, a. a. O., 776 f.).

23 J. Neu, Jealous Thoughts, a. a. O., 429: „It may be that precisely what we most value about certain relationships also makes them essentially nonreplicable and nonshareable, and hence leaves a place for jealousy.“

24 St. Cavell, Introduction, in: ders., Disowning Knowledge, a. a. O., 9.

25 W. Shakespeare, Othello, Stuttgart 1985 (alle Zitate nach dieser Ausgabe).

26 St. Cavell, Othello and the Stake of the Other, a. a. O., 141 (dt.: Der Anspruch der Vernunft, a. a. O., 785).

27 Vgl. J. Conant, On Bruns, on Cavell, in: Critical Inquiry, 17 (1991), 616-634: „This failure of acknowledgment is then interpreted by the philosopher in us (call him Othello) as a failure of knowledge. It is converted into an intellectual difficulty, so that its solution now appears to require further application of the intellect: a search for more and better knowledge." (631)

28 St. Cavell, Recounting Gains, Showing Losses. Reading The Winter's Tale, in: ders., Disowning Knowledge, a. a. O., 193-221, hier: 196 (dt.: ders., Gewinne und Verluste. Eine Lektüre des Wintermärchens, in: ders., Die Unheimlichkeit des Gewöhnlichen, a. a. O., 147-181, hier: 151).

29 Ebd., 195 (dt.: Gewinne und Verluste, a. a. O., 149).

30 W. Shakespeare, The Winter's Tale/Das Wintermärchen, Stuttgart 1987 (alle Zitate nach dieser Ausgabe).

31 St. Cavell, Recounting Gains, Showing Losses, a. a. O., 211 (dt.: Gewinne und Verluste, a. a. O., 169).

32 M. Nussbaum, Hiding from Humanity. Disgust, Shame, and the Law, Princeton 2004, $87 \mathrm{ff}$.

33 Vorausgesetzt ist, dass Ekel überhaupt als Emotion gefasst wird. Nicht alle Autoren teilen diese Auffassung, worauf hier aber nicht näher eingegangen werden kann.

34 W. Shakespeare, Coriolanus/Coriolan, Tübingen 2001 (alle Zitate nach dieser Ausgabe).

35 St. Cavell, Coriolanus and Interpretations of Politics, in: ders., Disowning Knowledge, a. a. O., 143-177, hier: $149 \mathrm{f}$.

36 Ebd., 165 (Hervorhebung von Cavell).

37 St. Cavell, Introduction, in: ders., Disowning Knowledge, a. a. O., 7; für die Interpretation der skeptischen Situation als eine der Nicht-Behauptung [non-claim] siehe auch St. Cavell, The Claim of Reason. Wittgenstein, 
Skepticism, Morality, and Tragedy, Oxford 1979, 217-221 (dt.: Der Anspruch der Vernunft, a. a. O., 363368).

38 Ders., Recounting Gains, Showing Losses, a. a. O., 206 (dt.: Gewinne und Verluste, a. a. O., 163).

39 A. Honneth, Verdinglichung, Frankfurt/M. 2005, 68.

40 St. Cavell, Knowing and Acknowledging, in: ders., Must We Mean What We Say? A Book of Essays, Cambridge 1976, 238-266, hier: 263 (dt.: ders., Wissen und Anerkennen, in: ders., Die Unheimlichkeit des Gewöhnlichen, a. a. O., 39-73, hier: 70).

41 Honneth ist sich darüber im Klaren, dass die Einnahme einer anerkennenden Haltung für Cavell nicht impliziert, „,em Anderen gegenüber stets eine wohlwollende, liebevolle Reaktion zu zeigen“(Verdinglichung, a. a. O., 59), aber es fällt ihm schwer, dieser interpretatorischen Einsicht in seinem eigenen Ansatz treu zu bleiben. Immer wieder neigt er dazu, Anerkennung als spezifische positive Reaktion auf den anderen zu fassen, obwohl für Cavell Anerkennung weder notwendig positiv noch notwendig an spezifische Reaktionen gebunden ist. Anteilnahme [sympathy] etwa ist für Cavell keine vorgängige Form der Anerkennung, sondern eine mögliche Reaktion auf den Schmerz des anderen. Ausbleibende Anteilnahme kann dann eine Form der Anerkennung sein, wenn sie auch als Reaktion auf den Schmerz des anderen gedeutet werden kann (,Dein Schmerz interessiert mich nicht!'). Ein solcher Anerkennungsbegriff ist nur schwer vereinbar mit dem von Honneth an vielen Orten entwickelten Begriff.

42 St. Cavell, Knowing and Acknowledging, a. a. O., 263 (dt.: Wissen und Anerkennen, a. a. O., 70).

43 Es ist nur konsequent, dass Cavell dort, wo er den Verdinglichungsbegriff explizit aufgreift (The Claim of Reason, a. a. O., 372 ff.; dt.: Der Anspruch der Vernunft, a. a. O., 591 ff.), Zweifel an der Annahme hegt, wir könnten Menschen ernsthaft als Dinge behandeln, solange wir in irgendeiner Form mit ihnen interagieren. Selbst der Sklavenhalter glaubt nicht, dass Sklaven keine Menschen sind, er sieht nur „bestimmte Menschen als Sklaven“ (375; dt. 597): ,Was er übersieht, ist nicht eigentlich etwas an Sklaven und auch nicht eigentlich etwas an Menschen. Er übersieht etwas an sich selbst oder vielmehr etwas an seiner Verbindung mit diesen Menschen, sozusagen an seiner inneren Beziehung zu ihnen.“

44 Vgl. St. Cavell, The Philosopher in American Life, in: ders., In Quest of the Ordinary. Lines of Skepticism and Romanticism, Chicago 1988, 3-26, hier: 8: „I do not propose the idea of acknowledging as an alternative to knowing but rather as an interpretation of it." Siehe auch ders., The Claim of Reason, a. a. O., 428 (dt.: Der Anspruch der Vernunft, a. a. O., 679): „In ,Knowing and Acknowledging ‘ habe ich behauptet, Anerkennung gehe über Wissen ,hinaus ' nicht in der Art der Kognition oder als kognitive Leistung, sondern weil ich aufgefordert bin (call upon me), dies Wissen in seinem Kern zu äußern (express), zu erkennen, was ich weiß, in seinem Lichte etwas zu tun, das, unterbliebe es, dieses Wissen ohne Ausdruck (without expression) zurückließe."

45 A. Honneth, Verdinglichung, a. a. O., 57.

46 Vgl. M. Hartmann, Gefühle. Wie die Wissenschaften sie erklären, Frankfurt/M. 2005, 163.

47 Seine eher hilflose Diskussion der Frage, ob der Zweifel ein männliches Phänomen ist, bestätigt diesen Punkt: St. Cavell, Introduction, in: ders., Disowning Knowledge, a. a. O., $16 \mathrm{f}$.

48 Ders., The Avoidance of Love, a. a. O., $94 \mathrm{f}$.

49 Hier gibt es im Übrigen eine komplizierte Nähe zu pragmatistischen Wahrnehmungstheorien. Cavell hat seine Nähe zum Pragmatismus stets geleugnet, um seine Nähe zum amerikanischen Transzendentalismus desto stärker hervorzuheben. Über die Quellen dieser verdrängten Verwandtschaft kann hier aus Platzgründen nichts gesagt werden.

50 In diesem Sinne rekonstruiert Wollheim Emotionen mithilfe eines „historical account“; siehe R. Wollheim, On the Emotions, New Haven 1999, 17 (dt.: ders., Emotionen. Eine Philosophie der Gefühle, München 2001, 33); siehe auch A. Baier, What Emotions Are About, in: Philosophical Perspectives, 4 (1990), 1-29, hier: 6: „Representation will come into emotion not primarily through the beliefs or evaluations directly implied in a current emotion [...] but in the way this emotional episode repeats and represents a series of past emotions of the same type."

51 Ch. Voss, Narrative Emotionen. Eine Untersuchung über Möglichkeiten und Grenzen philosophischer Emotionstheorien, Berlin 2004, 186.

52 Narratologische Theorien im engeren Sinne veranschaulichen ihre Begrifflichkeiten in der Regel mithilfe literarischer Beispiele. Entsprechend postulieren sie einen „,narrativen Akteur“, der im Rahmen einer Erzählung bestimmte Ereignisse wiedergibt (vgl. M. Bal, Narratology. Introduction to the Theory of Narrative, 
Toronto ${ }^{2}$ 1997). Narrative Emotionstheorien dagegen setzen den Interpreten der Emotion als narrativen Akteur und brauchen nicht zwangsläufig auf einen fiktiven Erzähler Bezug zu nehmen. Durch diese Verschiebung der Perspektive verliert der Begriff des Narrativen zweifellos an Kontur.

53 L. Daston, Die moralischen Ökonomien der Wissenschaft, in: dies., Wunder, Beweise und Tatsachen. Zur Geschichte der Rationalität, Frankfurt/M. 2001, 157-184, hier: 159.

54 B. Williams, Truth and Truthfulness. An Essay on Genealogy, Princeton 2002, 233 (dt.: ders., Wahrheit und Wahrhaftigkeit, Frankfurt/M. 2003, 345).

55 Ich stütze mich hier auf: R. Moran, Authority and Estrangement. An Essay on Self-Knowledge, Princeton 2001, 49: ,These two people may both be grateful, but they will undoubtedly feel and think and act differently in the expression of their gratitude, and we would expect the histories of their emotions to follow different courses. Any description of them which left out of account the differences in how they conceive of (their) gratitude would not simply be incomplete, but would be seriously misleading." 